\title{
Research Paper \\ Impact of Some Macroeconomic Variables on the Development of the Financial Sector A Model For Gulf Cooperation Council (GCC) States
}

Journal of

\section{TANMIYAT AL- RAFIDAIN}

\section{(TANRA)}

A scientific, quarterly, international, open access, and peer-reviewed journal

Vol. 40, No. 130

June 2021

(C) University of Mosul | College of Administration and Economics, Mosul, Iraq.

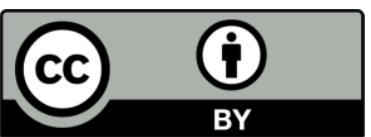

TANRA retains the copyright of published articles, which is released under a "Creative Commons Attribution License for CC-BY-4.0" enabling the unrestricted use, distribution, and reproduction of an article in any medium, provided that the original work is properly cited.

Citation: AL-Dabbgh, Nawar, AL-Iragi , Bashar A (2021). "Impact of Some Macroeconomic Variables on the Development of the Financial SectorAModelForGulf

Cooperation Council (GCC) States". TANMIYAT ALRAFIDAIN, 40 (130), ץ • ₹ -230, https://doi.org/

10.33899/tanra.2020.127995.10

40

P-ISSN: 1609-591X

e-ISSN: 2664-276X

tanmiyat.mosuljournals.com

\section{Nawar K. AL-Dabbagh ${ }^{1}$ Bashar A. AL-Iragi ${ }^{2}$}

$1 \& 2$ University of Mosul, College of Business and Economics, Department of Banking and Financial Sciences

Corresponding author: Nawar K. AL-Dabbagh, University of Mosul, College of Business and Economics, Department of Banking and Financial Sciences

Nawarkanaan2019@gmail.com

DOI: https://doi.org/ 10.33899/tanra.2020.127995.1040

Article History: Received: 12/8/2020; Revised: 6/9/2020; Accepted: 22/9/2020; Published: 1/6/2021.

\section{Abstract}

The research aims to provide a deep and comprehensive picture of the theoretical frameworks and empirical studies that dealt with diagnosing the impact of some macroeconomic variables on the development of the financial sector, in addition to exploring an objective quantitative model that can monitor the nature, size, and direction of this effect and explain its mechanism and channels of transmission. By adopting the methodology of aggregate regression models (PRM), fixed effect (FEM), and random effect (REM) that are based on the Balanced Panel Data, for the GCC countries for the period (2005-2017). The results of the assessment revealed the participation of the trade openness variable in its positive impact on the three indicators of financial sector development, $(B C)$ which expresses the bank credit granted to the private sector as a percentage of GDP, (TD) which indicates the bank's concentration (the percentage of bank assets that It is held by the first three banks) and (SPV) which reflects market fluctuations, while the inflation variable (INF) exerted a negative effect on $(B C),(T D)$ and (IS), finally, the growth variable (GRO), which negatively affected ( $B C)$

\section{Keywords}

Financial sector, financial development, economic growth, inflation, trade openness, balanced panel data methodology. 


\section{ورقة بحثية أثر بعض متغير ات الاقتصاد الكلي في تثمية القطاع المالي دول مجلس التعاون الخليجي أنموذجياً التئياد}

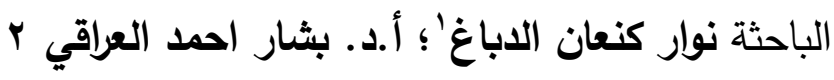

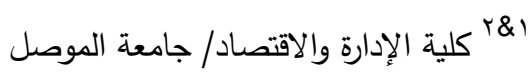

المؤلف المراسل الباحثة نوار كنعان الدباغ جامعة الموصل، الموصل، العراق، nawarkanaan2019@gmail.com.

DOI: https://doi.org/ 10.33899/tanra.2020.127995.1040

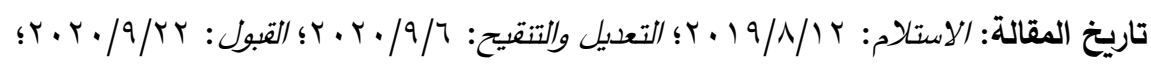

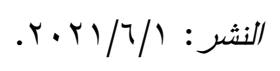

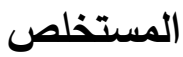

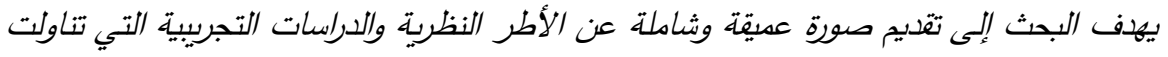

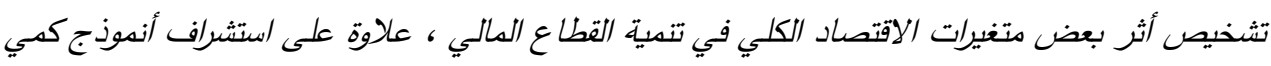

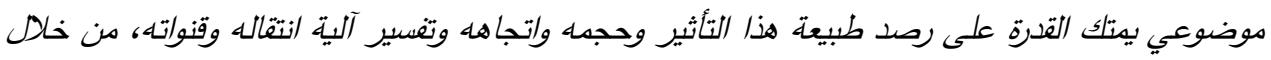

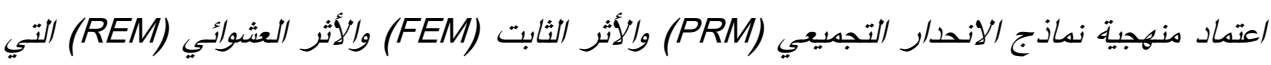

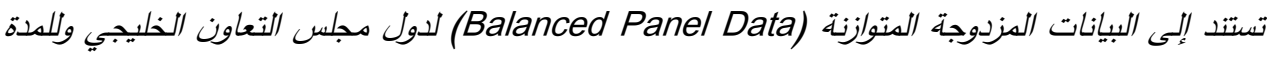

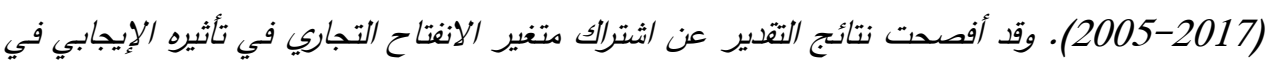

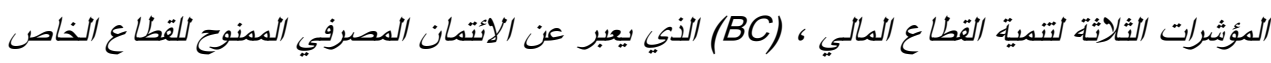

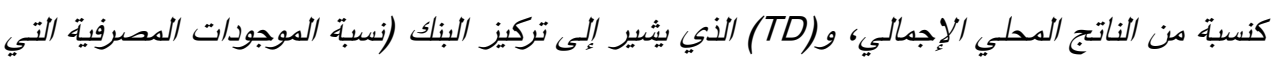

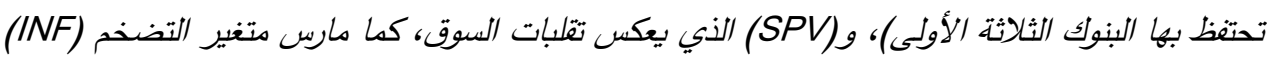

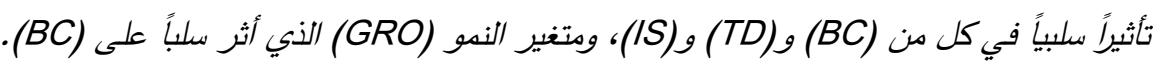

الكلمات الرئيسة

القطاع المالي، التنمية المالية، النمو الاقتصادي، التضخم، الانفتاح التجاري، منهجية البيانات (Blanced panel Data) المزدوجة المتوازنة

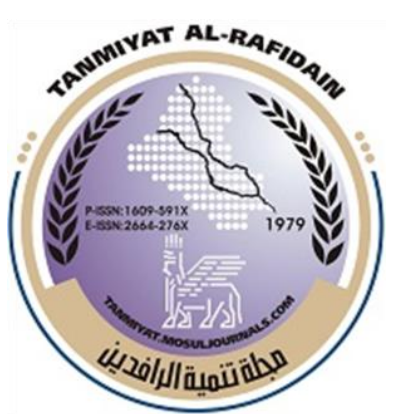

هبلة

\section{تنمية الرافدين}

(TANRA) دولية، مفتوحة الوصول، محكمة.

$$
\begin{aligned}
& \text { المجلد (•• )، العدد (•r ())، } \\
& \text { حزيران ا.r.r }
\end{aligned}
$$

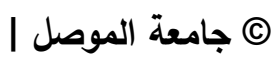
كلية الإدارة والاقتصاد، الموصل، العراق.

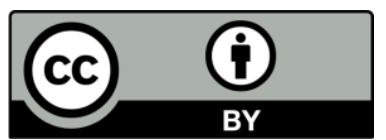

تحتفظ (TANRA) بحقوق الطبع والنشر للمقالات

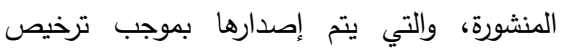

لـ (Creative Commons Attribution) (CC-BY-4.0)

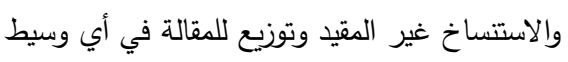
نقل، بشرط اقتباس العمل الأصلي بشكل صحيح.

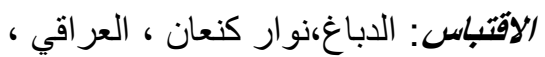

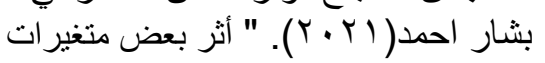
الاقتصاد الكلي في تتمية القطاع المالي التئي دول مجلس التعاون الخليجي أنموذجاد التهاء

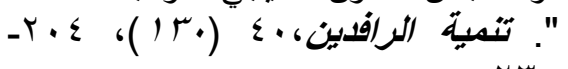
https://doi.org/ 10.33899/tanra.2020.127995.10 40

P-ISSN: 1609-591X e-ISSN: 2664-276X tanmiyat.mosuljournals.com 


\section{أثر بعض متغيرات الاقتصاد الكلي في تنمية القطاع .................}

الدباغ والعراقي

يؤدي القطاع المالي وبحسب ما تجمع عليه دعظم الأدبيات المالية والاقتصادية' دوراً بالغ الأهمية في اقتصاديات الدول، نظرا لروابطه الأمامية والخلفية مع الكثير من المتغيرات الاقتصادية، بما يجعل التقدم

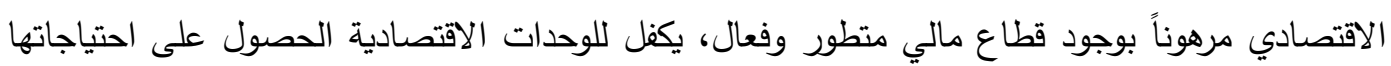
المالية وتعزيزها من خلال قدرته على تعبئة المدخرات وتوجيهها نحو الفرص الاستثمارية الأكثر انتاجية (ربحية) وإدارة وتوزيع المخاطر على من هم أكثر قدرة على احتمالها، وتوفير التسهيلات والترتيبات لتسوية المدفوعات، والحد من اللا تماثل المعلوماتي، فضلا عن تحفيز الوحدات والقطاعات الاقتصادية للدفع بها

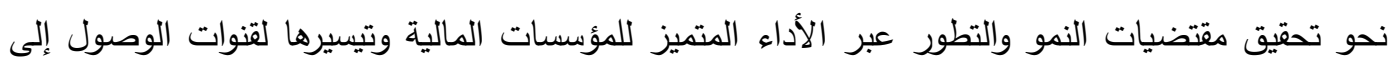
أدوات وخدمات مالية متتوعة وبتكلفة مقبولة، وكل ذلك يعمل على خلق آثار إيجابية على معدلات النمو والاستثمار في الاقتصاد الوطني، بما يكفل تحقيق مستوى معيشي أفضل لجميع أفراد المجتمع. • أهمية البحث

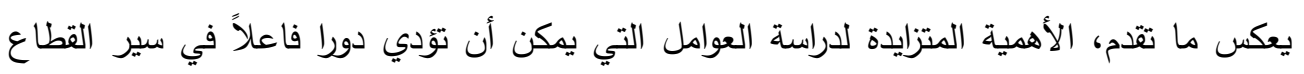

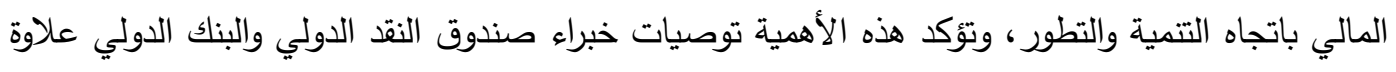
على الأطر النظرية والتجريبية بضرورة رصد وتثخيص تلك العوامل، ومنها النمو، والتضخم، والانفتاح التهاح

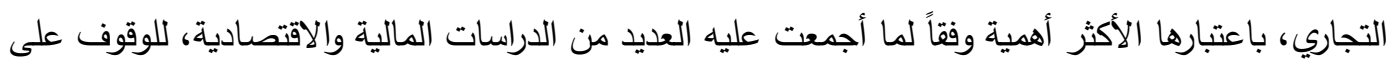
العوامل الإيجابية منها والتي تسهم في رفع مستوى تتمية القطاع المالي بهدف دعمها من جانب، وتحديد العوامل السلبية التي تعيق و/أو تمنع من تطوره والععل على معالجتها من جانب آخر ، باعتبارها الارضية

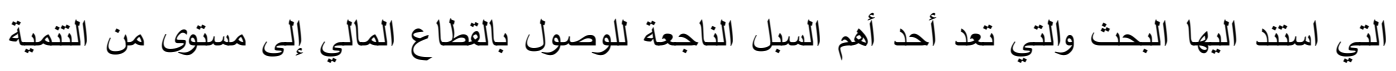

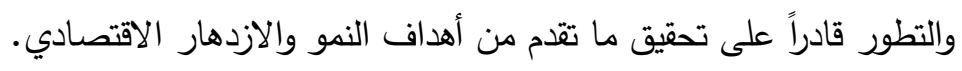
• مشكلة البحث.

تخضع مستويات التطور المالي في بلدان العالم ولا سيما النامية منها لمجموعة من العوامل والمحددات

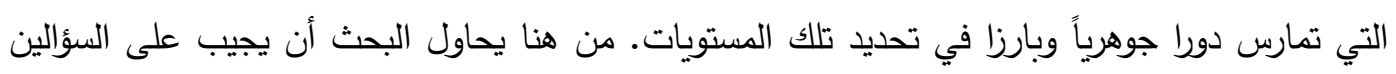

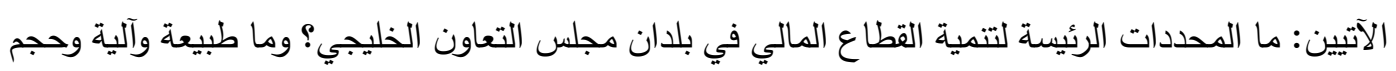
ذلك التأثير الذي يمكن أن يمارسه كل محدد منها؟ • هدف البحث.

سعت الدراسة إلى عرض الأدبيات الاقتصادية والمالية التي حددت مصفوفة العوامل الاقتصادية

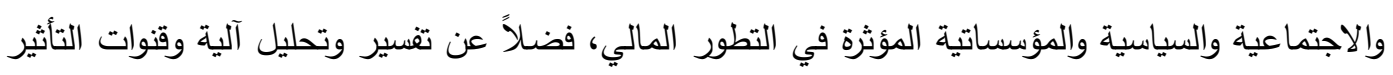

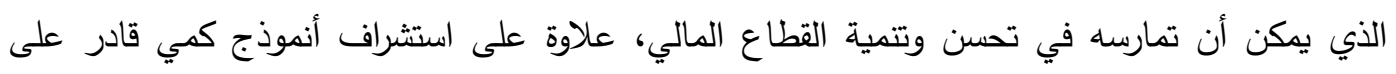

1. Christopoulos \& Tsionas (2004), King \& Levine (1993), Levine, Loayza \& Beck (2000), Calderon \& Liu (2003), Bayar, Gündüz \& Sezgin (2019) 


\section{أثر بعض متغيرات الاقتصاد الكلي في تنمية القطاع ..........} الدباغ والعراقي

تشخيص طبيعة واتجاه وحجم ذلك التأثير وبالتالي تسهم في تغذية وتفسير التفاوت والاختلاف بين البلدان في تطان مستوى تطور قطاعاتها المالية وتتميتها.

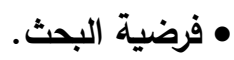
انطلق البحث من فرضيات أساسية مفادها الآتي:

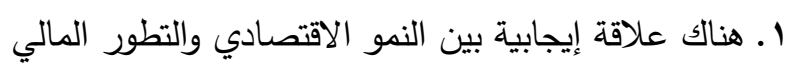

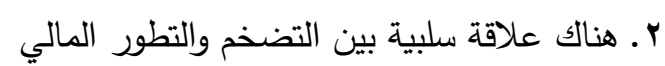

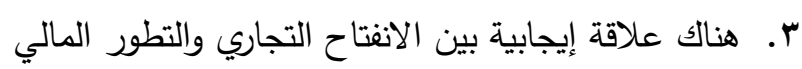
أولاً: الإطار النظري

أشارت معظم الأدبيات المتعلقة بدراسة تتمية القطاع المالي إلى أن العوامل الرئيسة المحددة له يمكن

$$
\text { أن تؤطر في الآتي: النمو الاقتصادي: }
$$

على الرغم من انعدام الرؤية الواضحة حول طبيعة واتجاه العلاقة السببية التي تربط بين نمو القطاع

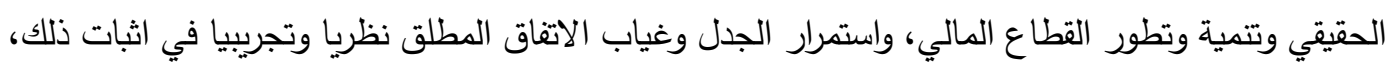

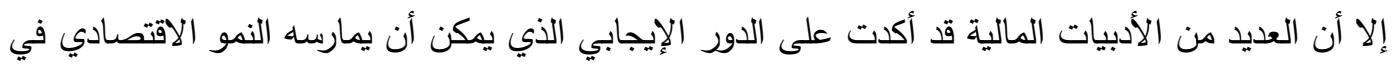
تطور القطاع المالي وتنميته. ويشير (1952) Robinson، إلى أن النمو الاقتصادي عادة ما يدفع بالقطاع الإنيات المالي، ونتيجة لقدرته على خلق طلب جديد وتحفيز الطلب الحاضر على الأدوات والخدمات المالية، باتجاه التتمية والتطور • وأيد Patrick (1966) ذلك بالقول، ومن خلال ما أطلق عليه بفرضية تتبع الطلب (DFH) (Demand Following Hypothesis)

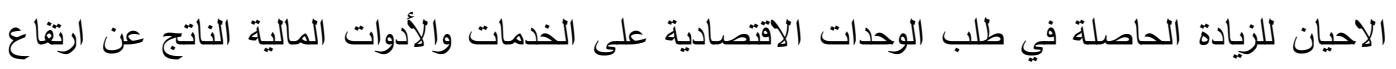
معدلات النمو الاقتصادي وتأثيراته الإيجابية في الدخل والاستثمار ، وبشكل أكبر عند ارتفاع مستويات التباين

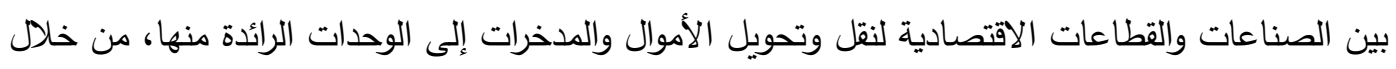

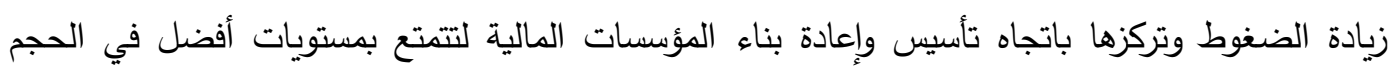

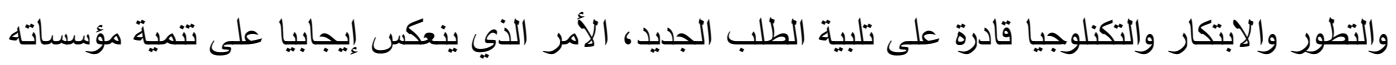

وتطور هيكليته وأدواته (Ehigiamusoe, Guptan, \& Narayanan,2019,3) (Ndalu,2017.40).

r و التضخم

لم تبتعد غالبية الآراء المطروحة في الأدبيات الاقتصادية والمالية عن عد المحافظة على معدلات

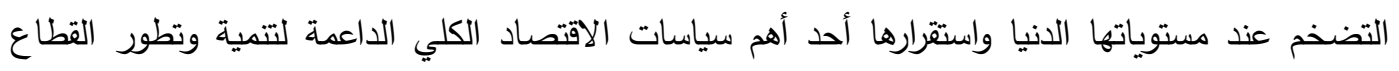

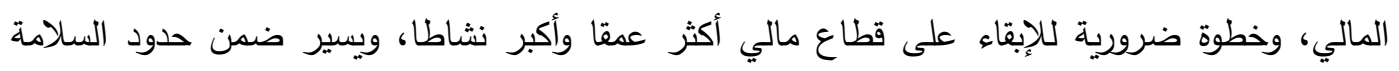
والاستقرار (Didier \& Schmukler,2014,4), (Bittencourt,2012,341-355). فالمعدلات العالية من التضخم يمكن أن تمارس تأثيرات غير مرغوبة في تتمية القطاع المالي ومن خلال عدة قنوات. فهي تعمل فلالترات 
Huybens \& Smith,1999,283-( على خلق حالة من عدم اليقين تتسبب في ارتفاع نسب المخاطرة 315), (Ozturk \& Karagoz,2012,81-87)، (Boyd et al.,2001,221-228)، وتسهم في توليد بعض عيوب السوق، وتساعد على إزالة ما يعرف بتماثل المعلومات (Asymmetric Information)، وسيادة المخاطر الاخلاقية (Moral Hazard)، وتضعف من قدرة المؤسسات المالية على التمييز بين إمكانيات المقترضين على الالتزام المالي، كما تخفض من معدل العائد الحقيقي على النقود والموجودات وترفع من احتكاكات سوق الائتمان (Credit Market Friction) (Zermeñoa et al.,2018,94-129)، وتساعد على تآكل القيم الحقيقية لمدخرات الوحدات الاقتصادية ولعوائدها المالية التي تقف كحواجز مانعة

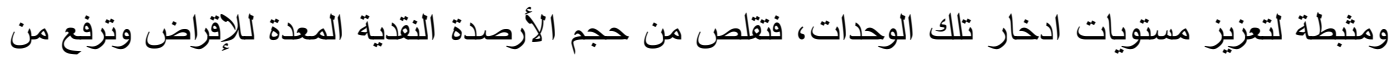
أسعار الفائدة فتلجأ الوحدات الاقتصادية وبهدف مواجهة المخاطر المحتملة الناتجة عن كل ذلك إلى الاحتفاظ بمحافظ استثمارية عالية السيولة (Bittencourt,2008,3)، كما تقف معدلات التضخم العالية عوائق أمام

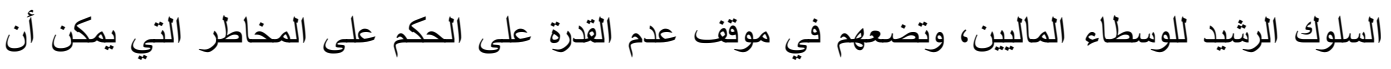
تتعرض إليها القرارات الاستثمارية وبشكل كاف Baum et al.2006,289-304),(Baum et) al.,2009,87-89). إن جميع التأثيرات السابقة لمعدلات التضخم تتسبب مجتمعة بتجريد القطاع المالي من أدواته الكفوءة والفعالة في تعبئة الموارد المالية وإعادة تخصيصها باتجاه الاستثمارات الأفضل كفاءة والأكثر انتاجية، وهو ما ينعكس سلبا على أداء وهيكلية القطاع المالي من مصارف وأسواق مالية، فيعيق تنميتها

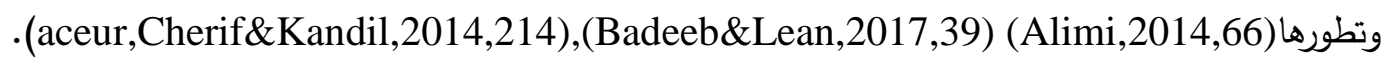

r. الانفتاح التجاري (Trade Openness) يثكل جانبا الطلب والعرض في السوق المالي Supply and Demand Sides of the الاساس النظري الذي اعتمدت عليه معظم الأدبيات المالية في تفسير التأثير الإيجابي للانفتاح التجاري في تتمية القطاع المالي. ففي الجزء المتعلق بجانب العرض يرى Braun \& Raddatz

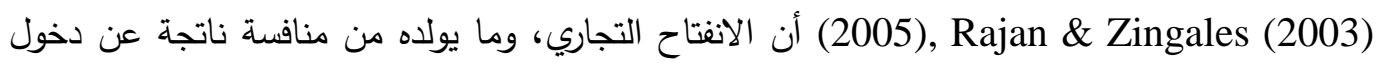

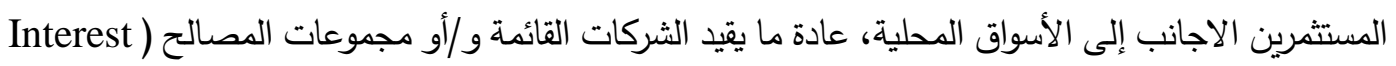

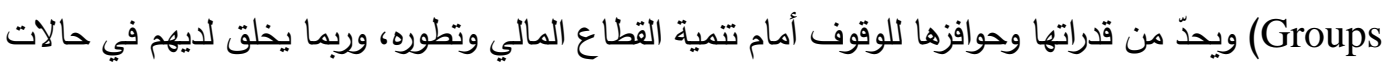

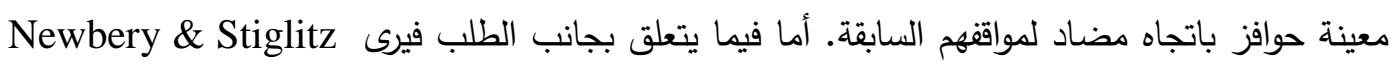
Svaleryd \& Vlachos (2000,2005) Beck (2002) (1984)

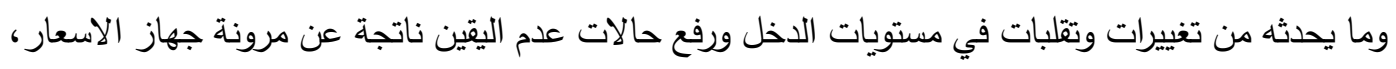
علاوة على المنافسة الاجنبية وإمكانية الخضوع للصدمات الخارجية، قد يحفز الطلب على المنتجات والخدمات

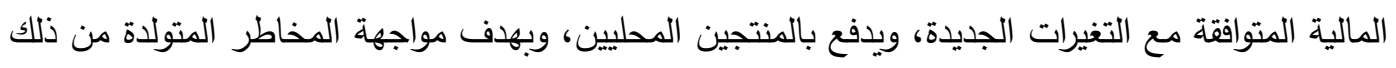
إلى اللجوء إلى التتويع الذي يتطلب توفير التمويل اللازم والمدعوم بسياسات وخدمات تأمين مناسبة، الأمر 


\section{أثر بعض متغيرات الاقتصاد الكلي في تنمية القطاع ..................} الدباغ والعراقي

Ho \& ( الذي يدفع بالقائمين على القطاع المالي ولمواجهة ذلك إلى دفعه باتجاه التطور والتتمية .(Bernard,2018,4 ثانيا: مراجعة الأدبيات ذات العلاقة

لم يكن تثخيص العوامل المؤثرة في تتمية وتطور القطاع المالي والوقوف على طبيعة واتجاه هذا

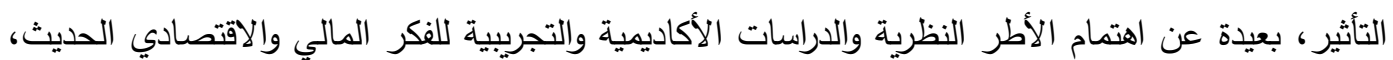

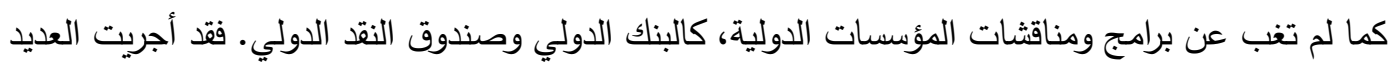
من البحوث الدراسات التي تناولت تلك العوامل بصورها المفردة أو المجموعة ولعينات ودول مختلفة، يمكن

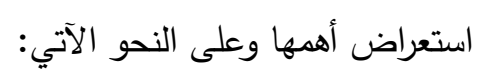

اهتمت دراسة Nejad (2010) بمحددات التمية المالية وتقييم آثار السياسات المالية القدعية على

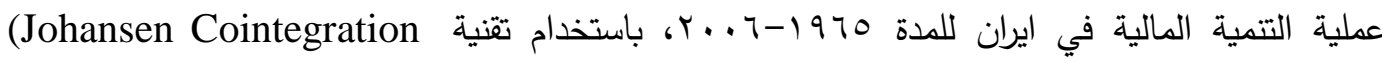
لبيانات السلسلة الزمنية. وأكدت نتائج العمل التجريبي أن كلاً من الانفتاح التجاري والادخار

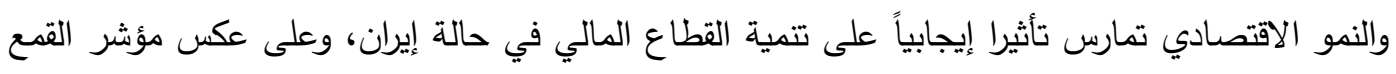

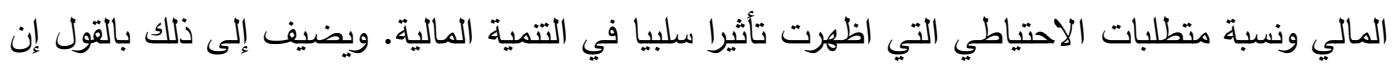

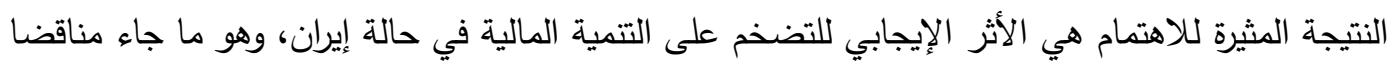
للمنطق الاقتصادي، الا أنه يتماشى مع الرأي القائل بوجود معدل تضخم حرج، لإيجى يمكن أن يثجع النشاط الحقيقي، ويعزز التتمية المالية بدلاً من عرقلتها.

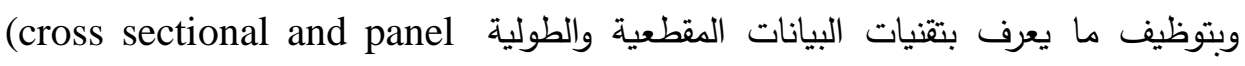
dوبناء تشخيص كمي لأثر الانفتاح التجاري والمالي ومعدلات نمو الناتج المحلي الإجمالي (المتغيرات

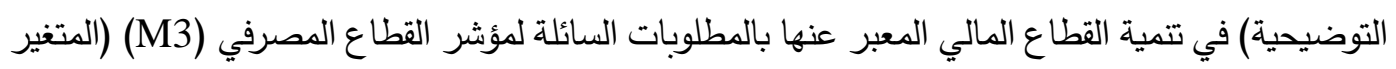

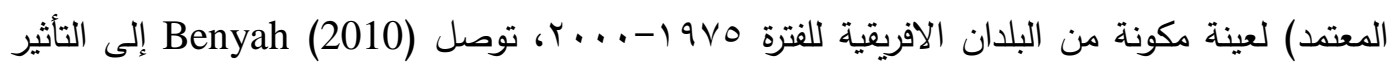

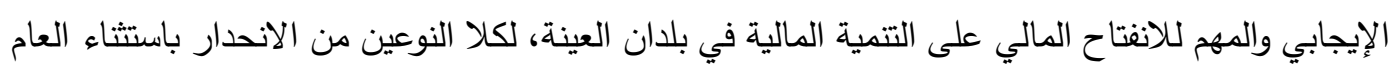

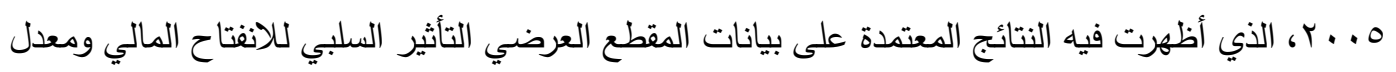

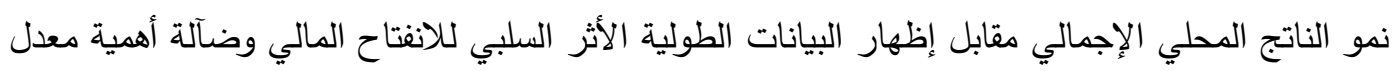

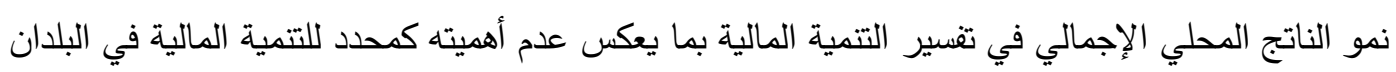
الأفريقية . نو

في دراسة (2013) Takyi \& Obeng حول محددات التتمية المالية في غانا وباستخدام منهجية

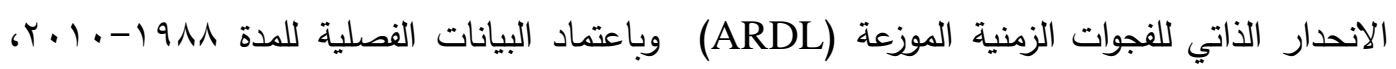

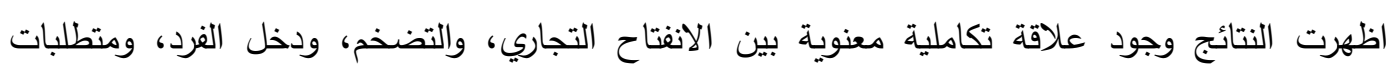
الاحتياطي والاقتراض الحكومي، علاوة على الدور المهم الذي يمكن أن يؤديه الانفتاح التجاري ودخل الفرد ولئح

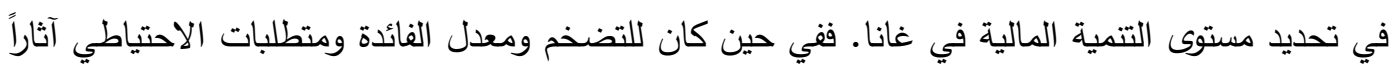


سلبية وذا دلالة إحصائية على التتمية المالية في المدى القصير والطويل، فثل الاقراض الحكومي في تأكيد

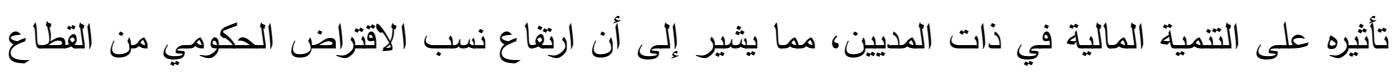

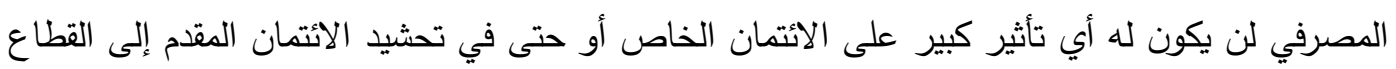

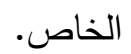

وركزت دراسة (2014) Badeeb \& Lean على تحليل محددات التتمية المالية في اليمن وبالاعتماد

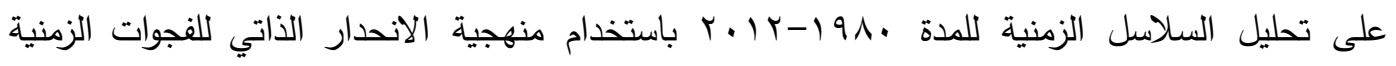
الموزعة ARDL مع تضمين أكثر العوامل المحتملة تأثيراً على وتيرة التتمية المالية فيها، وهي النمو

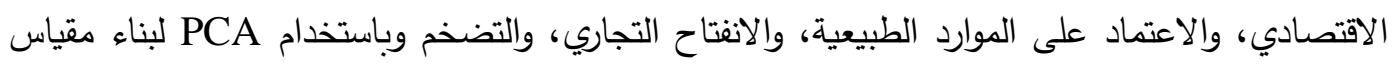

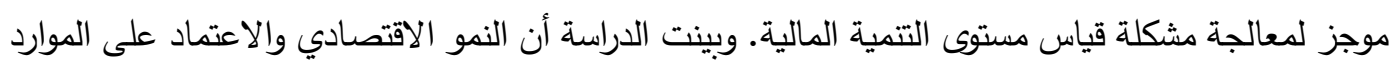
الطبيعية والانفتاح التجاري والتضخم هي المحددات الأكثر أهمية في التأثير على التتمية المالية في اليمن.

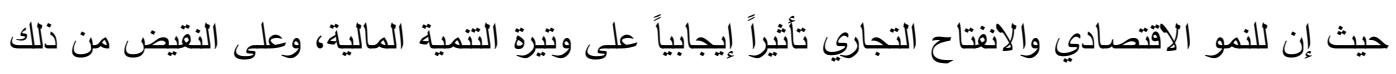

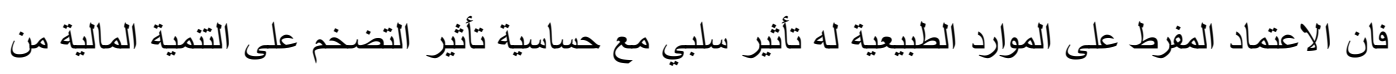

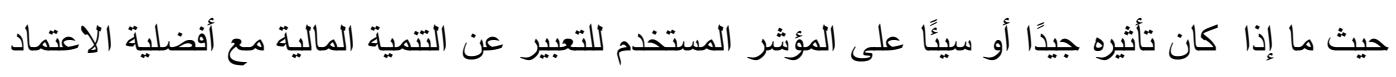
على مؤشر وأحد لالتقاط معظم خصائص التتمية المالية من حيث العمق والكئ الكفاءة.

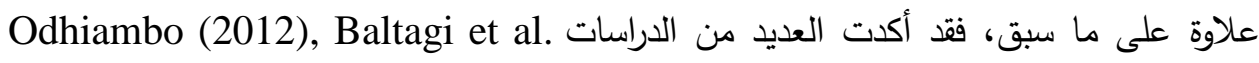
(2009), Arestis \& Demetriades (1996), Law \& Habibullah (2009) ,Hurlin \& Venet الاحأ (2008), Gozgor (2015),Peia \& Roszbach (2015),Zang \& Kim (2007)، على التأثير الإيجابي للنمو الاقتصادي في رفع معدلات التنمية المالية، وعدت مستويات الدخل أحد المحددات الهامة

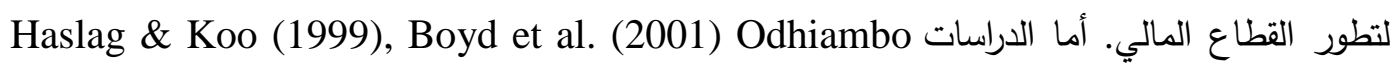
(2012) Bittencourt (2008), Naceur, Cherif \& Kandil (2014)) Alimi (2014) على الآثار السلبية الضارة لمعدلات التضخم المرتفعة على تتمية القطاع المالي وتطوره، بينما أثرت الدراسات Do \& Levchenko (2007), Law (2008,2009), Iyke et al. (2016)

$$
\text { من التنمية المالية بدرجة الانفتاح التجاري }
$$

ثالثاً: المنهجية التجريبية (Empirical Methodology)

في ضوء ما تقدم من دراسات سابقة للباحثين الاقتصاديين والماليين، واستتادا إلى الأطر النظرية

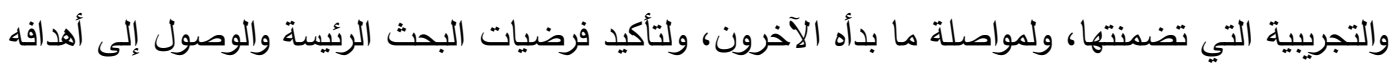
الاساسية، وبهدف تصميم أنموذج كمي لأثر واتجاه علاقة مصفوفة متغيرات الدراسة (المتغيرات التوضيحية المتمثلة: بالنمو الاقتصادي، والتضخم، والانفتاح التجاري) في تتمية القطاع المالي ومؤشراته (المتغير المعتمد)

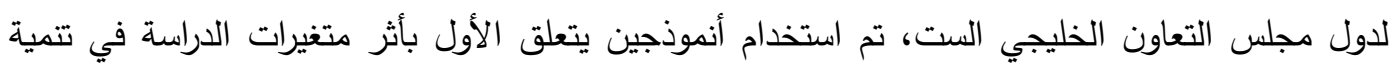

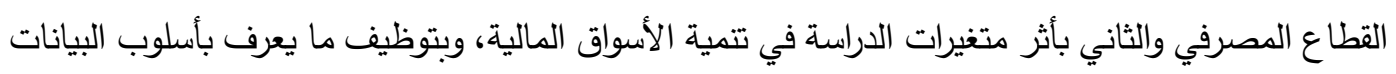




\section{أثر بعض متغيرات الاقتصاد الكلي في تنمية القطاع ..................} الدباغ والعراقي

المزدوجة المتوازنة (Balanced Panel Data) وللفترة (2005-2017) وبواقع عدد مشاهدات السلسلة

$$
\text { الزمنية (V^) مشاهدة }
$$

بهدف توحيد مصادر البيانات وتجنب تباينها واختلافها اشتملت مصادر البيانات لدول مجلس التعاون

الخليجي (عينة الدراسة) على ثلاثة أجزاء، الاول مأخوذ من قاعدة بيانات التمية المالية العالمية Global) Financial Development Database) مؤشرات التتمية المالية. في حين يستند الثاني والثالث على التوالي إلى قاعدة بيانات البنك الدولي والمعنية بمؤشرات التتمية العالمية (World Development Indicators)، وقاعدة بيانات صندوق النقد الدولي الخاصة بالإحصاءات المالية الدولية (International Financial Statistics) للوصول إلى بيانات

$$
\begin{aligned}
& \text { المتغيرات التفسيرية (النمو الاقتصادي، والتضخم، والانفتاح التجاري) . }
\end{aligned}
$$

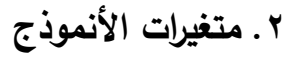

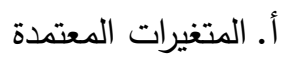

انطلاقاً من البحوث والدراسات التجريبية مثل (2019) Svirydzenka Gupta \& Mahakud (2016)، (2012) . Čihák et al) والمستتدة لآراء خبراء البنك الدولي وصندوق النقد الدولي في تمييز مؤشرات تتمية القطاع المالي، تم اعتماد مؤشرين للتعبير عن تتمية الأسواق المالية، ومؤشرين للتعبير عن لاء تتمية القطاع المصرفي، والتي عدت أكثرها شيوعا واستخداما من قبل تلك الدراسات وكما يأتي:

$$
\text { 1. ـ مؤشرات القطاع المصرفي }
$$

الائتمان المصرفي الممنوح للقطاع الخاص كنسبة من الناتج المحلي الإجمالي (BC): ويعد من

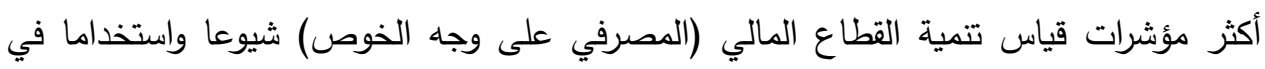
الدراسات الاقتصادية والمالية، نظرا لقدرته على التعبير عن حجم الوساطة المالية ومستوى كفاءة التهات تخصيص الموارد التي يمكن أن يمارسها القطاع المالي من الاقتصاد وبشكل خاص فئسير في البلدان النامية ذات المستويات غير المتدنية من تشوهات السوق(Asiama \& Mobolaji,2011,5) ، فارتفاع قيمته عادة ما تدلل على تمتع القطاع المالي بمستويات عالية من الفاعلية والتطور . (Ross et al., 2000,31-77) .(Asiama \& Mobolaji,2011,5)

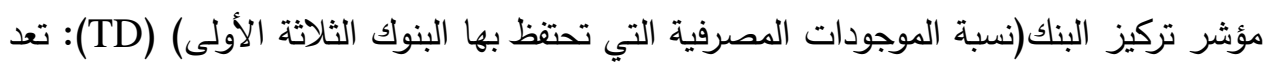
نسبة الموجودات المصرفية التي تحتفظ بها البنوك الثلاثة الأولى من أهم المؤشرات المستخدمة

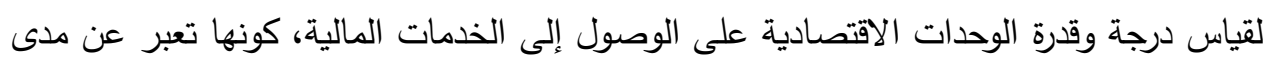

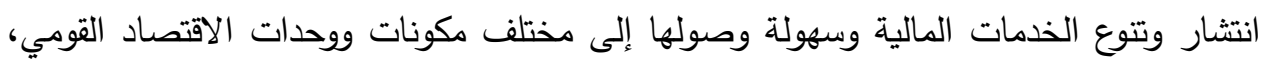
فارتفاع نسبته يعكس وجود عدد قليل من المؤسسات المالية التي تحوز على نسبة عالية من موارد

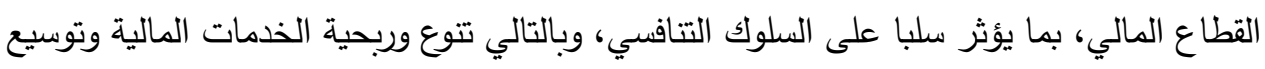


قاعدة الزبائن بما يصب في تقويض التتمية المالية. (Bara, (Svirydzenka, 2016,10)

(Jacolin \& Chauvet, 2016,2) Mugano \& Roux, 22017, 152)

r ب مؤشرات الأسواق المالية

• سندات الدين الدولية (إصدارات صافية) / الناتج المحلي الإجمالي (IS): والذي يقاس بإجمالي عدد

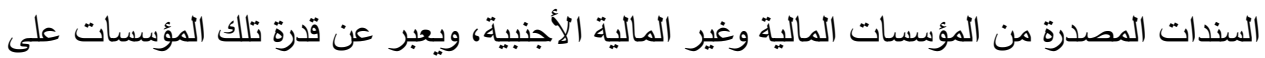

الوصول إلى الأسواق المالية Svirydzenka (2016) Setiawan (2015). مؤشر تقلبات السوق (SPV): ويعد المؤشر الأكثر استخداما وشيوعا للتعبير عن الاستقرار المالي،

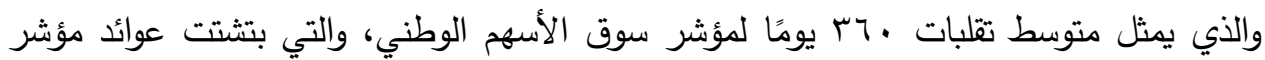

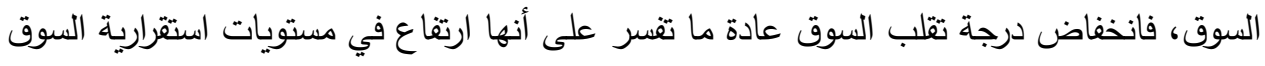

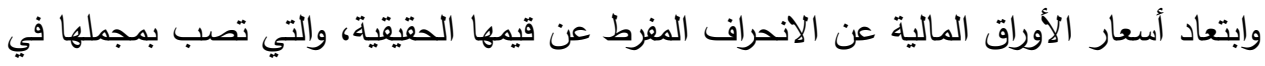
صالح تتمية وتطور الأسواق المالية، ومن ثم القطاع المالي الذي تعد الأسواق المالية جزءًا مهما من مكوناته (Manolescu\& Manolescu,2017,6)، (Zervos,1998,541)

$$
\text { أ. المتغيرات المستقلة (التوضيحية): }
$$

تم تصنيف المتغيرات المستقلة وتأثيراتها المتوقعة على النحو الآتي:

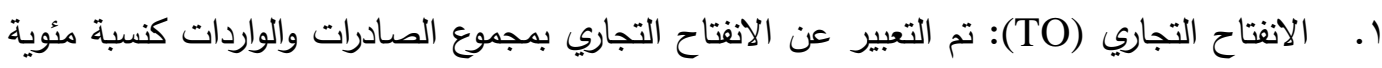
من الناتج المحلي الإجمالي، باعتباره المؤشر الأكثر شيوعا واستخداما في غالبية التبارية الدراسات التجريبية.

.(Ho \& Bernard,2018,6), (Huang,2010,165)

r. النمو الاقتصادي (GDP): ويعبر عنه بمعدل التغير في حصة الفرد من الناتج المحلي الإجمالي

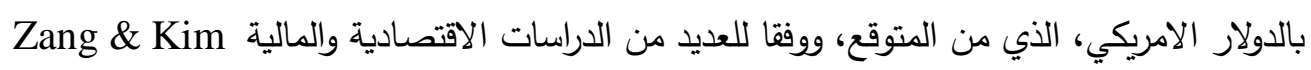
(2007) Arestis \& Demetriades (1996), Baltagi et al. (2009), Law \& Habibullah , (2009), Odhiambo (2012), Gozgor (2015),Peia \& Roszbach (2015), إيجابيا في تطور وتتمية القطاع المالية.

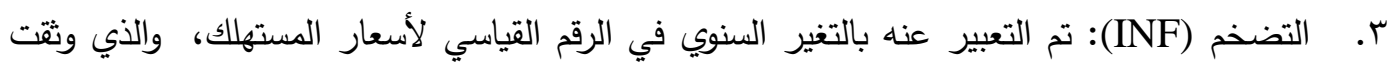
Boyd et al. (2001), Odhiambo (2012), Bittencourt (2008), معظم الدراسات التجريبية (Ben \& Kandil (2014)، الآثار السلبية لارتفاع معدلاته على تتمية القطاع المالي وتطوره.

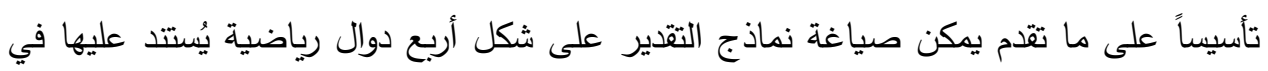

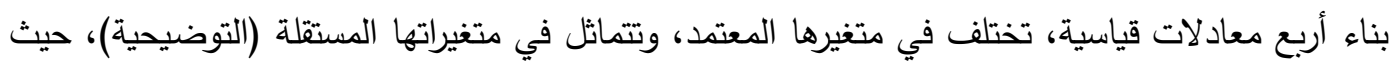
تعكس المعادلة الأولى أثر الانفتاح التجاري و النمو الاقتصادي والتضخم في مؤشر تتمية القطاع المالي ونئي

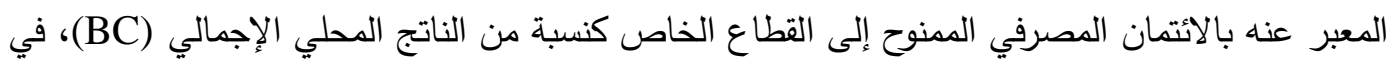

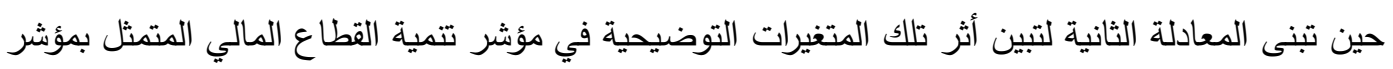


تركيز البنك(نسبة الموجودات المصرفية التي تحتفظ بها البنوك الثلاثة الأولى) (TD) بينما يؤشر الأنموذج الثالث أثر المتغيرات التوضيحية الثلاثة في مؤشر تتمية القطاع المالي المتمثل بـ (صافي الإصدارات): سندات الدين الدولية - جميع جهات الإصدار إلى الناتج المحلي الإجمالي بالدولار الأمريكي (IS) واخيراً توضح المعادلة الرابعة أثر التغيرات التوضيحية المعتمدة في النماذج السابقة في مؤشر تتمية القطاع المالي الإني

$$
\begin{gathered}
\text { المعبر عنه بمؤشر تقلبات السوق (SPV)، وكما يأتي: } 1 \text { (SP }) \\
\text { (1)BC }=F\{T R A, G R O, I N F\} \\
\text { (2)TD }=F\{T R A, G R O, I N F\} \\
\text { (3)IS }=F\{T R A, G R O, I N F\} \\
\text { (4)SPV }=F\{T R A, G R O, I N F\}
\end{gathered}
$$

(1) $B C=\beta_{0}+\beta_{1} T R A+\beta_{2} G R O+\beta_{3} I N F+\varepsilon_{i}$

(2)TD $=\beta_{0}+\beta_{1} T R A+\beta_{2} G R O+\beta_{3} I N F+\varepsilon_{i}$

(3)IS $=\beta_{0}+\beta_{1} T R A+\beta_{2} G R O+\beta_{3} I N F+\varepsilon_{i}$

(4) $S P V=\beta_{0}+\beta_{1} T R A+\beta_{2} G R O+\beta_{3} I N F+\varepsilon_{i}$

\section{r. منهجية تقدير الأنموذج.}

تكتسب البيانات المزدوجة (Panel Data) اهتماماً بالغاً في الآونة الاخيرة وبشكل خاص في لالإده الدراسات الاقتصادية والمالية، لما تتسم به من قدرة على الدمج بين خصائص كل من البيانات المقطعية

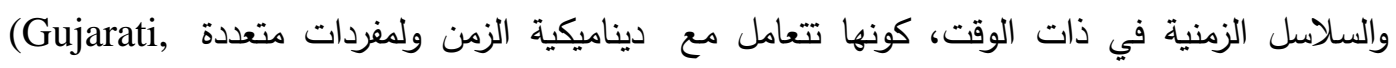

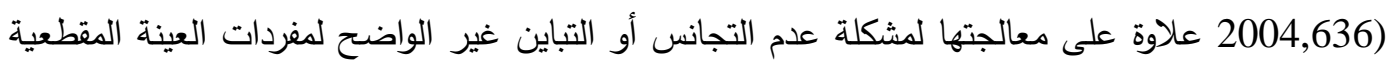
ومن ثم الابتعاد عن الوقوع في تقديرات متحيزة وغير متسقة (Reyna, 2007, 2). ويتم التعامل مع البيانات

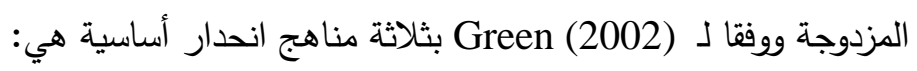
أ. أنموذج الانحدار التجميعي (Pooled Regression Model) (PRM)

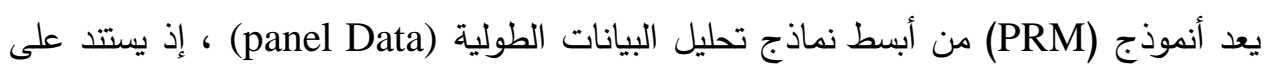

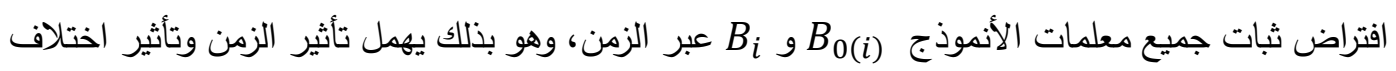
الخصائص الفردية في الأنموذج، بتجاهل طبيعة البيانات الطولية (Hiestand,2005,43) والتعامل معهات كسلاسل زمنية (Time Series)، حيث يعمل على ترتيب قيم المتغيرات المستقلة والمتغير المعتمد بشكل

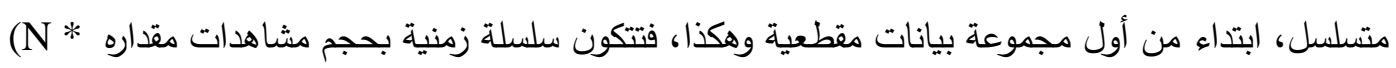

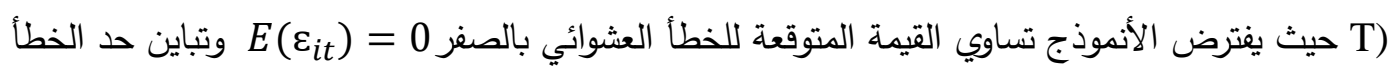
العشوائي ثابت الحصول على تقديرات تتصف بالاتساق (Consistent) والكفاءة (Efficient) شرط ثبات الأثر الفردي

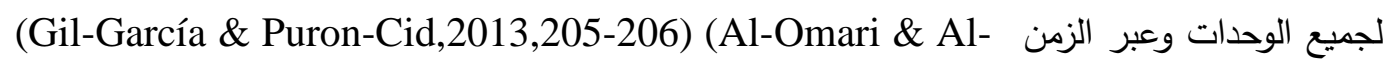
Hait, 2016, 883) ويتخذ أنموذج الانحدار التجميعي الصيغة الآتية : 


$$
\begin{array}{r}
Y_{i t}=\alpha_{i}+\sum_{i=1}^{k} B_{j} X_{j(i t)}+\varepsilon_{i t} \\
i=1,2, \ldots \ldots \ldots, N \quad t=1,2, \ldots \ldots \ldots .
\end{array}
$$

ب. أنموذج الأثر الثابت (Fixed Effect Model) (FEM)

يستند أنموذج الأثر الثابت (FEM) على افتراض وجود اختلافات غير مشاهدة وغير متجانسة

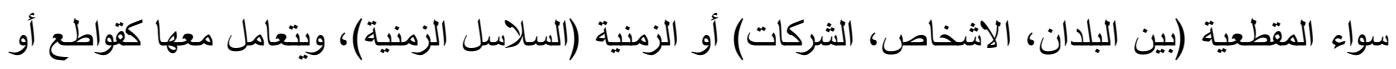

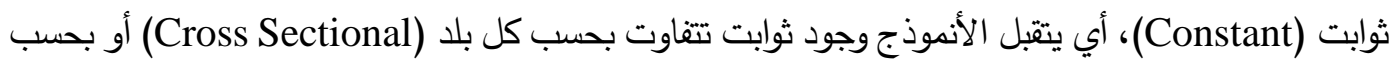
كل فترة زمنية (Time series) بهدف استكثاف الآثار غير المشاهدة والمؤثرة في المتغير المعتمد، ولتقدير بودير هذه الثوابت تستخدم طريقة المربعات الصغرى للمتغيرات الوهمية (Least-Squares Dummy (LSDV) والتي تحتوي على متغيرات وهمية بعدد (n-1) لتمثيل الدول و Variable Model) وفي ضوء ما سبق يعكس هذا الأنموذج الاختلافات والفروق بين الوحدات المقطعية للعينة. وتظهر الصيغة ولئة

$$
\begin{aligned}
& \text { الرياضية للأنموذج على النحو الآتي: } \\
& Y_{i t}=\alpha_{i}+\sum_{i=1}^{k} B_{j} X_{j(i t)}+\varepsilon_{i t} \\
& i=1,2, \ldots \ldots, N \quad t=1,2, \ldots \ldots, T \text { (3) } \\
& \alpha_{i}=\alpha_{0}+u_{i}
\end{aligned}
$$

(Clark et ، $\varepsilon_{i t ~} \sim N\left(0, \sigma^{2}\right)$ إذ تتوزع الاخطاء العشوائية طبيعيا حول الوسط بمتوسط صفر، وتباين ثابه

(Al-Iraqi, 2018, 71-104) al.,2010,7-8)

ت. أنموذج الأثر العشوائي(Random Effect Model) (REM) على خلاف أنموذج الأثر الثابت (FEM)، يتعامل أنموذج الأثر العشوائي (REM) مع الآثار المقطعية

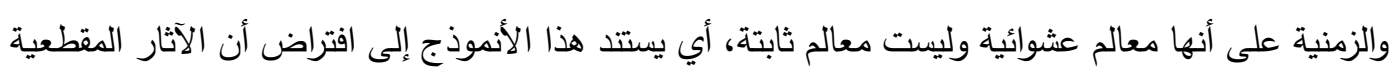

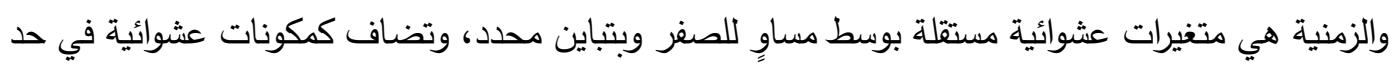

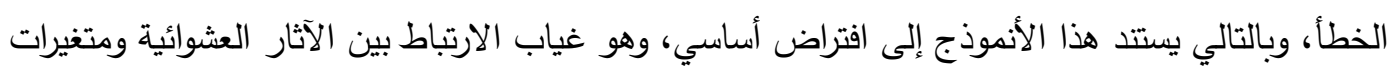

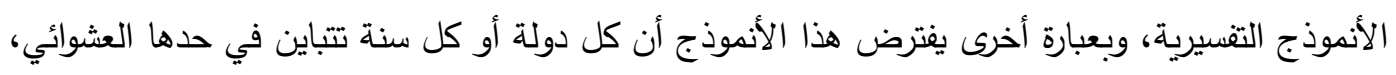

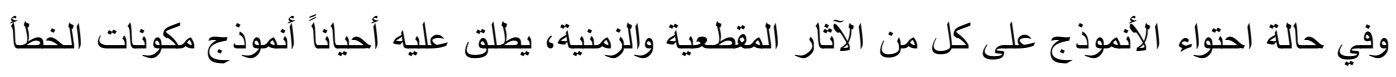

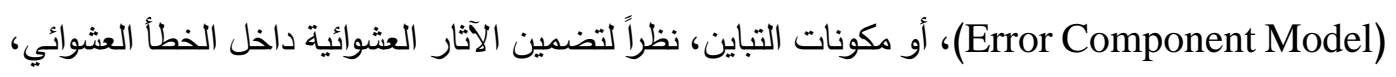
وبالتالي احتوائه على مركبين للخطأ، وبذلك يتخذ أنموذج الأثر العشوائي (REM) الصيغة التينة الآتية:

$$
\begin{gathered}
Y_{i t}=\alpha_{i}+\sum_{i=1}^{k} B_{j} X_{j(i t)}+\epsilon_{i t} \\
i=1,2, \ldots . ., N \quad t=1,2, \ldots ., T \\
\epsilon_{i t}=u_{i}+\varepsilon_{i t}
\end{gathered}
$$




\section{أثر بعض متغيرات الاقتصاد الكلي في تنمية القطاع ..........} الدباغ والعراقي

بافتراض أن حد الخطأ المركب $\epsilon_{i t}$ يمتلك خصائص الاخطاء العشوائية الاخرى نفسها من حيث

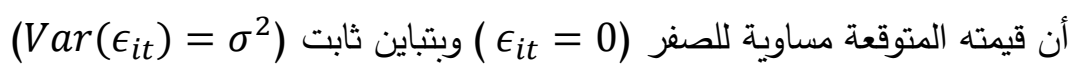

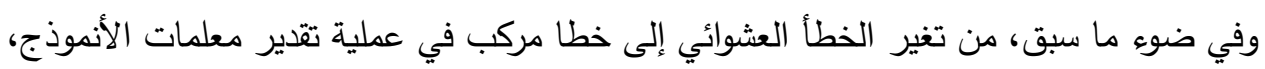

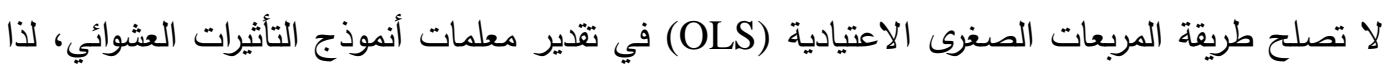

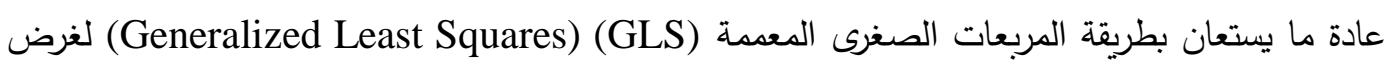

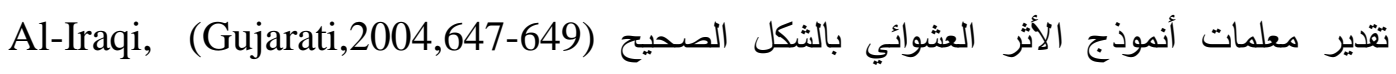
(Bashar, 2017, 445-474)

ولاختيار الأنموذج الأكثر ملاءمة من ضمن نماذج البيانات المزدوجة (Panel Data) الثلاثة

$$
\text { السابقة، يتم إجراء اختبارين احصائيين تثخيصيين يتمان عبر مرحلتين: }
$$

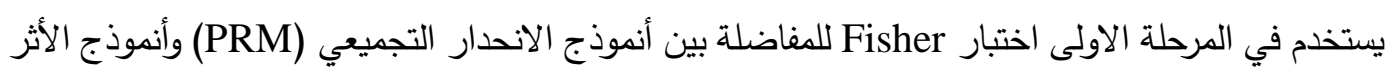

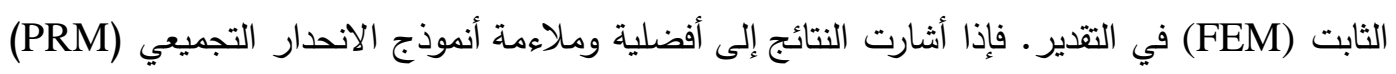

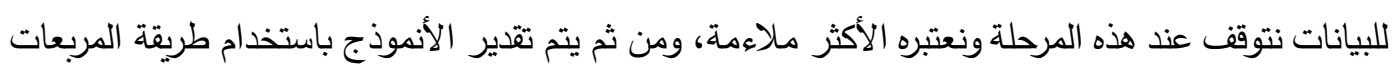

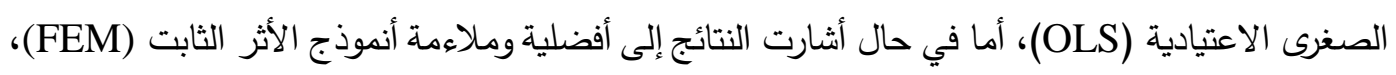

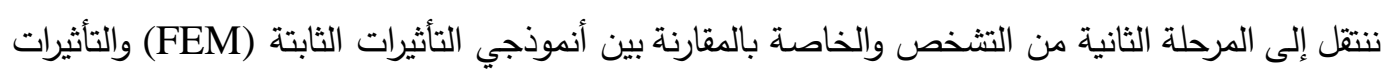
العشوائية(REM) بتطبيق اختبار (1978) Hausman (Clark et al.,2010,25-27). ـ ـ التوصيف الاحصائي لمتغيرات الدراسة

تُستخدم الإحصائيات الوصفية لاستعراض السمات الأساسية لبيانات متغيرات الدراسة والمعروضة

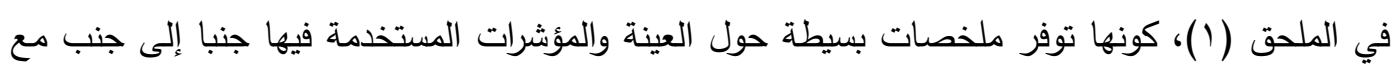
تحليل الرسوم البيانية، وبالتالي فإنها عادة ما تثكل أساس التحليل الكمي للبيانات، حيث تقوم الإحصائيات

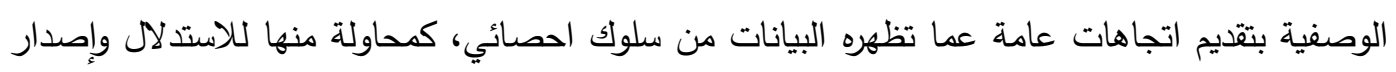

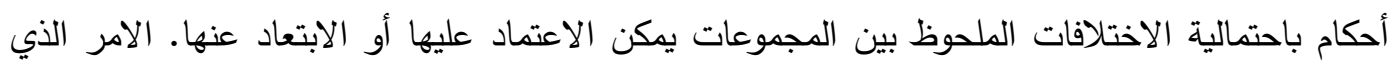

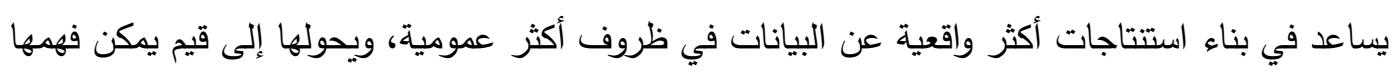
واستيعاب سلوكياتها بصورة أكثر سهولة. وعادة ما يثتمل التوصيف الاحصائي الأدوات الآتية:

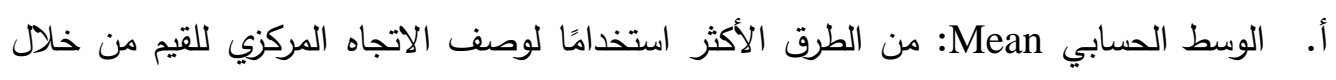
المتوسط العام لها.

ب. الوسيط Median: هو القيمة الوسطى (أو متوسط القيمتين الوسطتين) للسلسلة عندما يتم ترتيب

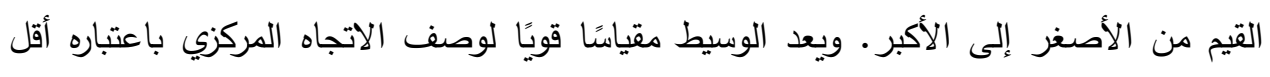
حساسية للقيم المتطرفة من المتوسط. ت. أعلى وأدنى قيمة Minimum, Maximum: هي القيم القصوى والدنيا للسلسلة في عينة الدراسة. وتعبر عن وصف الاتجاه المركزي لسير المتغيرات. 


\section{أثر بعض متغيرات الاقتصاد الكلي في تنمية القطاع ...............}

الدباغ والعراقي

ث. الانحراف المعياري.Std. Dev : هو تقدير أكثر دقة وتفصيلًا للتشتت، حيث يوضح العلاقة بين

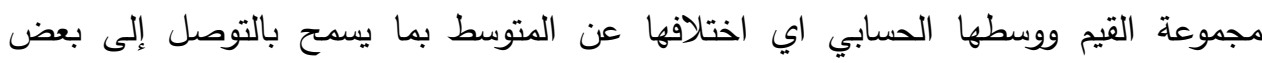
الاستتاجات حول توزيع القيم من حيث كونه توزيعاً طبيعيا (على شكل جرس أو قريباً منه).

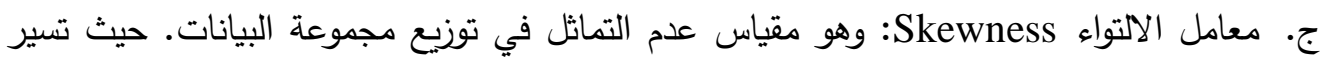
بعض القيم في نطاق قياسي، بينما يسير بعضها الآخر أعلى أو أقل بكثير من ذلك ذلك النطاق.

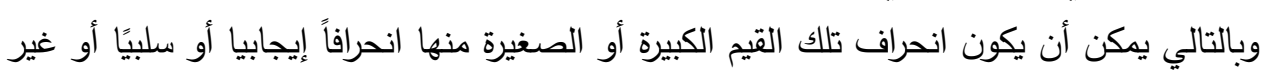
محدد. ح. معامل التفلطح Kurtosis: يصف معامل التفلطح حدة التوزيع، فالتوزيع بمعامل تفلطح كبير له

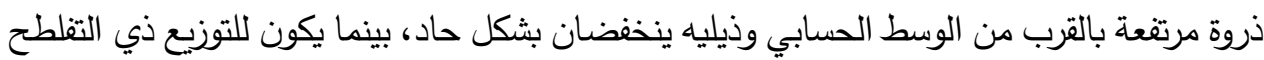

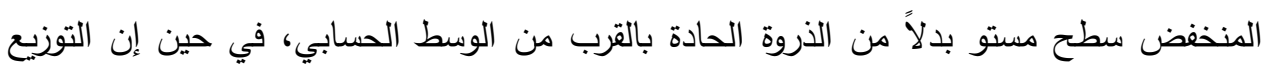
الموحد سيكون حالة متطرفة ونادرة الحدوث.

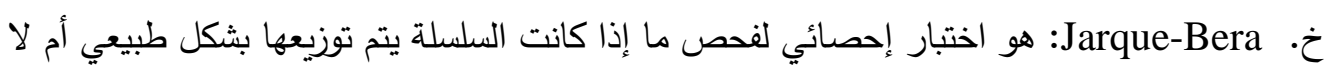

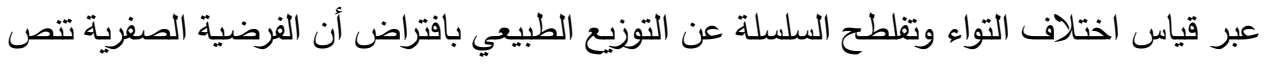

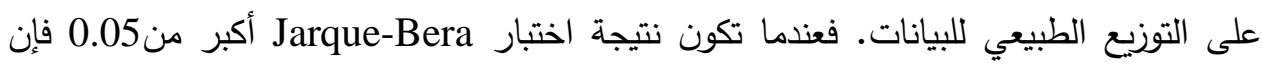
البيانات تتبع التوزيع الطبيعي (Hayek, 2018,46) د. الاحتمالية Probability: يعبر عن احتمالية التوزيع من حيث كونه منفصلاً أو مستمراً لمتغير وأحد أو أكثر ، أي يصف احتمالية أن وجود قيمة معينة (منفصلة) أو مجموعة من القيم (مستمرة)

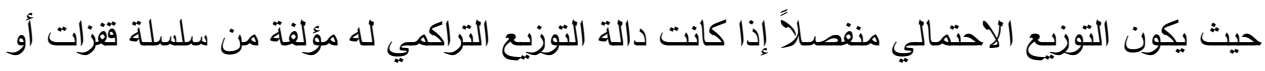

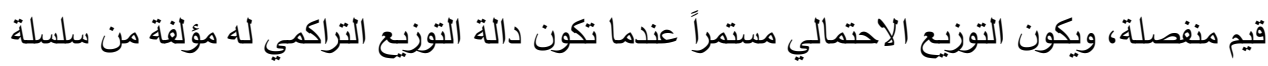
أو مجموعة قيم مستمرة. (DeCoursey, 2003, 85)

ه ـ تقدير النماذج وتحليل ومناقشة النتائج. للوصول إلى نتائج وتقديرات لأثر الانفتاح التجاري، والنمو الاقتصادي والتضخم على تتمية القطاع

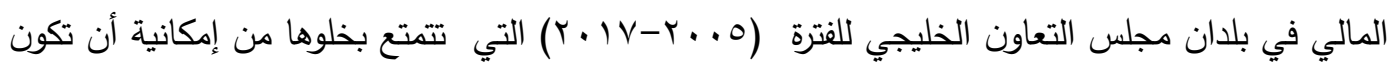
قيمها زائفة أو مضللة من خلال التأكد من استقرارية البيانات الخاصة بمتغيرات الدراسة وبصيغتها الخطية، والتي عكستها الرسوم البيانية في الملحق (Y)، وعدم احتوائها على جذر الوحدة، تم اعتماد اختبار لـ (LLC) (2002)

Levin, A., Lin, C.F., Chu, C. (2002) .Unit root tests in panel data: Asymptotic . and Finite Sample Properties. Journal of Econometrics,108:124. 
الدراسة إلا عند الفرق الأول، باستثناء متغيري التضخم (INF)، وصافي الاصدارات (IS) الذين أثبتا

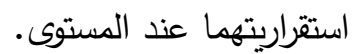

بناءً على ما تقدم من معطيات، وباعتماد نتائج اختبار Chow-Test (1960) حيث يقوم باختبار

$$
\text { الفرضية العدمية H0 }
$$

والتي تثير إلى قبول أنموذج الانحدار التجميعي PRM مقابل الفرضية البديلة H1 التي تثير إلى التى

قبول أنموذج الآثار الثابتة FEM وبعد اجراء الاختبار عكست نتائجه التي عرضت في الجدول ( (1)، امكانية ترجيح أنموذج الأثر الثابت (FEM) وعده الأكثر ملاءمة وأفضلية مقارنة بأنموذج الانحدار التجميعي

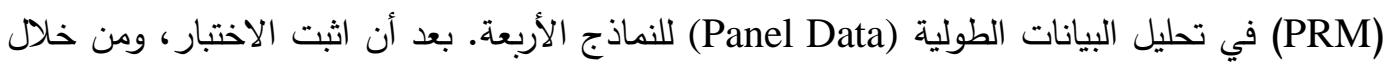

انخفاض قيمة.prob عن الـ 0.05 ، معنويته الاحصائية وترجيحه لقبول فرضية العدم.

الجدول (1) Chow-Test للنماذج الأربعة

\begin{tabular}{|c|c|c|c|}
\hline \multicolumn{4}{|c|}{$\begin{array}{l}\text { Redundant Fixed Effects Tests } \\
\text { Equation: Untitled } \\
\text { Test cross-section fixed effects }\end{array}$} \\
\hline \multicolumn{4}{|c|}{ للأنموذج الاول BC } \\
\hline Effects Test & Statistic & d.f. & Prob. \\
\hline Cross-section F & 11.026306 & $(5,70)$ & 0.0000 \\
\hline Cross-section Chi-square & 45.307874 & 5 & 0.0000 \\
\hline \multicolumn{4}{|c|}{ للأنموذج الثاني Chow-Test TD } \\
\hline Effects Test & Statistic & d.f. & Prob. \\
\hline Cross-section F & 187.401042 & $(5,70)$ & 0.0000 \\
\hline Cross-section Chi-square & 207.966784 & 5 & 0.0000 \\
\hline \multicolumn{4}{|c|}{ للأنموذج الثالث Chow-Test IS } \\
\hline Effects Test & Statistic & d.f. & Prob. \\
\hline Cross-section F & 2.878591 & $(5,70)$ & 0.0202 \\
\hline Cross-section Chi-square & 14.585117 & 5 & 0.0123 \\
\hline \multicolumn{4}{|c|}{ SPV للأنموذج الرابع SPV-Test } \\
\hline Effects Test & Statistic & d.f. & Prob. \\
\hline Cross-section F & 7.754908 & $(5,71)$ & 0.0000 \\
\hline Cross-section Chi-square & 33.988403 & 5 & 0.0000 \\
\hline
\end{tabular}

Eviews10 • • الجدول من إعداد الباحثين بالاعتماد على مخرجات برنامج

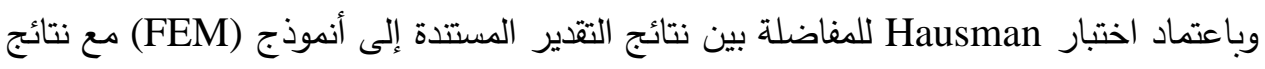
أنموذج (REM) للنماذج الأربعة، إذ يقوم باختبار الفرضية العدمية H0 والتي تثير إلى قبول أنموذج الأثر

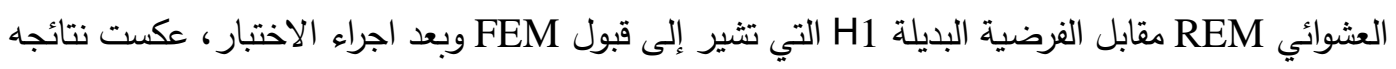
المثبتة في الجدول (2)، قبول الفرضية البديلة وما تفترضه من عدم اتساق النتائج المقدرة وفقا لمنهجية

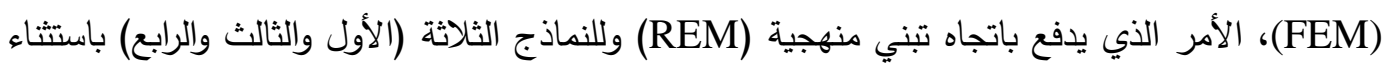




\section{أثر بعض متغيرات الاقتصاد الكلي في تنمية القطاع \\ الدباغ والعراقي}

الأنموذج الثاني الذي يفترض قبول فرضية العدم وما تعكسه من أفضلية واتساق النتائج الدقدرة وفقاً

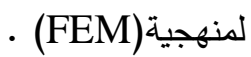

\section{الجدول (2) Hausman للنماذج الأربعة}

\begin{tabular}{|c|c|c|c|}
\hline \multicolumn{4}{|c|}{$\begin{array}{c}\text { Correlated Random Effects - Hausman Test } \\
\text { Equation: Untitled } \\
\text { Test cross-section random effects }\end{array}$} \\
\hline \multicolumn{4}{|c|}{ للأنموذج الأولHausman|ختبار } \\
\hline Test Summary & Summary Chi-Sq. Statisti & Chi-Sq. d.f & Prob. \\
\hline Cross-section random & 0.274961 & 2 & 0.8716 \\
\hline \multicolumn{4}{|c|}{ للأنموذج الثاني Hausman اختبار } \\
\hline Test Summary & Summary Chi-Sq. Statisti & Chi-Sq. d.f & Prob. \\
\hline Cross-section random & 0.267947 & 2 & 0.8746 \\
\hline \multicolumn{4}{|c|}{ للأنموذج الثالث Hausman اختبار } \\
\hline Test Summary & Summary Chi-Sq. Statisti & Chi-Sq. d.f & Prob. \\
\hline Cross-section random & 10.016099 & 2 & 0.0067 \\
\hline \multicolumn{4}{|c|}{ للأنموذج الرابع Hausman اختبار } \\
\hline Test Summary & Summary Chi-Sq. Statisti & Chi-Sq. d.f & Prob. \\
\hline Cross-section random & 0.056869 & 1 & 0.8115 \\
\hline
\end{tabular}

Eviews10 • • ل الجدول من اعداد الباحثين بالاعتماد على مخرجات برنامج

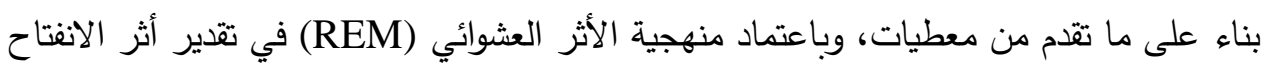
التجاري (TRA)، التضخم (INF)، والنمو الاقتصادي (GRO) في مؤشرات التتمية المالية المعبر عنها في

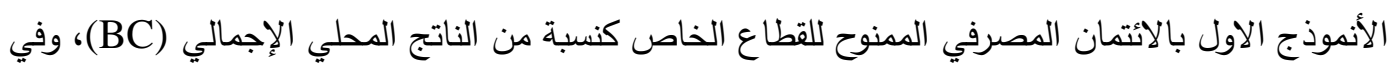
الأنموذج الثاني بتركيز البنك(نسبة الموجودات المصرفية التي تحتفظ بها البنوك الثلاثة الأولى) (TD) وفي الأني الأنموذج الثالث بـ(صافي الإصدارات): سندات الدين الدولية - جميع جهات الإصدار إلى الناتج المحلي الإجمالي بالدولار الأمريكي (IS) وفي الأنموذج الرابع بمؤشر تقلبات السوق (SPV)، والمدرجة نتائجها في

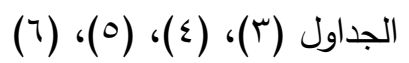

TANMIYAT AL-RAFIDAIN (P-ISSN: 1609-591X; E-ISSN: 2664-276X) تنمية الرافدين 
الجدول (3)أثر المتفيرات التوضيحية في مؤشرات تنمية القطاع المالي الأنموذج الاول أثر الانفتاح

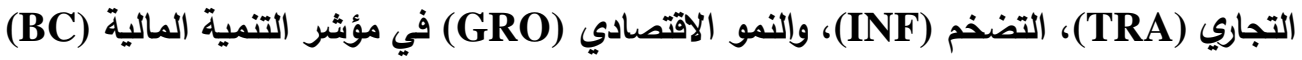

\begin{tabular}{|c|c|c|c|c|}
\hline \multicolumn{5}{|c|}{$\begin{array}{c}\text { Method: Panel EGLS (Cross-section random effects) } \\
\text { Sample: } 20052017 \\
\text { Periods included: } 13 \\
\text { Cross-sections included: } 6 \\
\text { Total panel (balanced) observations: } 78 \\
\text { Swamy and Arora estimator of component variances }\end{array}$} \\
\hline Variable & Coefficient & Std. Error & t-Statistic & Prob. \\
\hline GRO & -1.396401 & 0.326233 & -4.280384 & 0.0001 \\
\hline INF & -0.827043 & 0.417786 & -1.979585 & 0.0514 \\
\hline $\mathrm{C}$ & 66.50275 & 5.892297 & 11.28639 & 0.0000 \\
\hline \multicolumn{5}{|c|}{ Weighted Statistics } \\
\hline R-squared & 0.301995 & \multicolumn{2}{|c|}{ F-statistic } & 16.22454 \\
\hline Adjusted R-squared & 0.283382 & \multicolumn{2}{|c|}{ Prob. (F-statistic) } & 0.000001 \\
\hline Durbin-Watson stat & 0.673971 & & & \\
\hline
\end{tabular}

Eviews10 الجدول من عمل الباحثين بالاعتماد على مخرجات برنامج

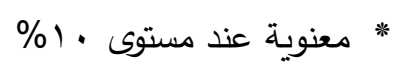

الجدول (4)أثر المتغيرات التوضيحية في مؤشرات تنمية القطاع المالي للأنموذج الثاني أثر الانفتاح

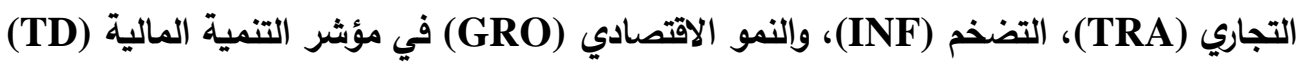

\begin{tabular}{|c|c|c|c|c|}
\hline \multicolumn{5}{|c|}{$\begin{array}{c}\text { Method: Panel Least Squares } \\
\text { Sample: } 20052017 \\
\text { Periods included: } 13 \\
\text { Cross-sections included: } 6 \\
\text { Total panel (balanced) observations: } 78\end{array}$} \\
\hline Variable & Coefficient & Std. Error & t-Statistic & Prob. \\
\hline INF & -0.220965 & 0.109522 & -2.017541 & 0.0475 \\
\hline TRA & 6.917182 & 2.704196 & 2.557944 & 0.0127 \\
\hline $\mathrm{C}$ & 64.55567 & 3.156381 & 20.45243 & 0.0000 \\
\hline \multicolumn{5}{|c|}{ Weighted Statistics } \\
\hline R-squared & 0.931170 & \multicolumn{2}{|c|}{ F-statistic } & 135.2858 \\
\hline Adjusted R-squared & 0.924287 & \multicolumn{2}{|c|}{ Prob. (F-statistic) } & 0.000000 \\
\hline Durbin-Watson stat & 0.630382 & & & \\
\hline
\end{tabular}

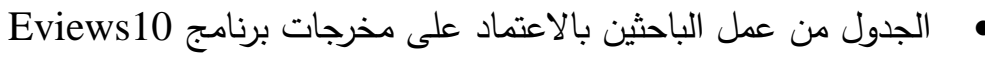

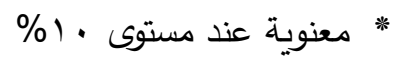

TANMIYAT AL-RAFIDAIN (P-ISSN: 1609-591X; E-ISSN: 2664-276X) تنمية الرافدين 
الجدول (5)أثر المتغيرات التوضيحية في مؤشرات تنمية القطاع المالي الأنموذج الثالث أثر الانفتاح

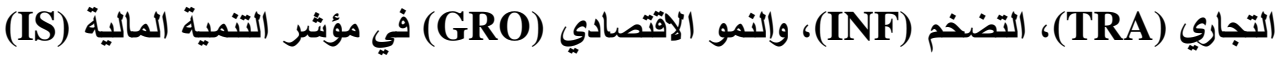

\begin{tabular}{|c|c|c|c|c|}
\hline \multicolumn{5}{|c|}{ Method: Panel EGLS (Cross-section random effects) } \\
Sample: 2005 2017 \\
Periods included: 13 \\
Cross-sections included: 6 \\
Total panel (balanced) observations: 78 \\
Swamy and Arora estimator of component variances \\
\hline Variable & Coefficient & Std. Error & t-Statistic & Prob. \\
\hline INF & -0.141959 & 0.055993 & -2.535308 & 0.0133 \\
\hline TRA & 0.027094 & 0.681236 & 0.039771 & 0.9684 \\
\hline C & 1.554138 & 0.848649 & 1.831309 & 0.0710 \\
\hline \multicolumn{5}{|c|}{ Weighted Statistics } \\
\hline R-squared & 0.072814 & F-statistic & 2.944969 \\
\hline Adjusted R-squared & 0.048089 & Prob. (F-statistic) & 0.058716 \\
\hline Durbin-Watson stat & 1.612638 & \multicolumn{3}{c|}{} \\
\hline
\end{tabular}

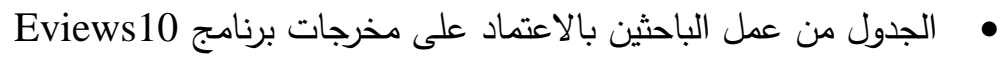

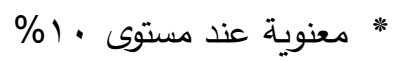

الجدول (6) أثر المتغيرات التوضيحية في مؤشرات تنمية القطاع المالي الأنموذج الرابع أثر الانفتاح

التجاري (TRA)، التضخم (INF)، والنمو الاقتصادي (GRO) في مؤشر التنمية المالية (SPV)

Method: Panel EGLS (Cross-section random effects)

Sample: 20052017

Periods included: 13

Cross-sections included: 6

Total panel (balanced) observations: 78

Swamy and Arora estimator of component variances

\begin{tabular}{|c|c|c|c|c|}
\hline Variable & Coefficient & Std. Error & t-Statistic & Prob. \\
\hline TRA & -11.63943 & 4.651678 & -2.502201 & 0.0145 \\
\hline C & 30.19643 & 5.810824 & 5.196584 & 0.0000 \\
\hline \multicolumn{5}{|c|}{ Weighted Statistics } \\
\hline R-squared & 0.076994 & F-statistic & 6.339681 \\
\hline Adjusted R-squared & 0.064849 & Prob. (F-statistic) & 0.013911 \\
\hline Durbin-Watson stat & 0.777986 & \multicolumn{1}{c}{} \\
\hline
\end{tabular}

Eviews10 الجدول من عمل الباحثين بالاعتماد على مخرجات برنامج

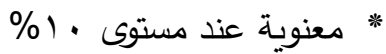

$$
\begin{aligned}
& \text { يمكن تشخيص ما يأتي: }
\end{aligned}
$$

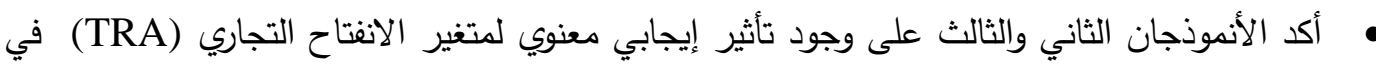

مؤشري التتمية المالية (TD), (IS) وبمعاملات بلغت (6.917182)، (0.027094)، على التوالي،

TANMIYAT AL-RAFIDAIN (P-ISSN: 1609-591X; E-ISSN: 2664-276X) تنمية الرافدين 


\section{أثر بعض متغيرات الاقتصاد الكلي في تنمية القطاع ..........}

الدباغ والعراقي

وهو ما جاء متوافقا مع الأطر النظرية والدراسات التجريبية التي أكدت على قدرة الانفتاح التجاري على تعزيز تتمية القطاع المالي. أكدت النماذج الثلاثة وجود تأثير سلبي معنوي لمتغير التضخم (INF) في مؤشرات التتمية المالية

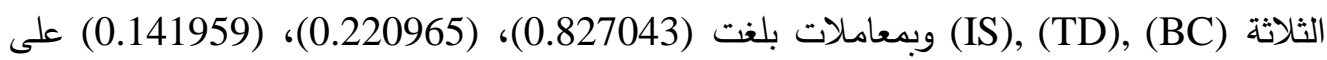

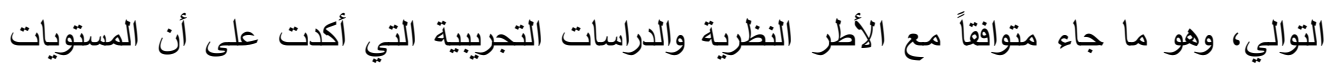
المرتفعة من التضخم تقوض تتمية القطاع المالي. جاء الأثر السلبي لمؤشر نمو الناتج المحلي الإجمالي (GRO) في مؤشر التتمية المالية (BC) مناقضاً للمنطق الاقتصادي، والذي يشير إلى انخفاض معدل نمو الناتج المحلي الإجمالي يعمل على رفع الإنى

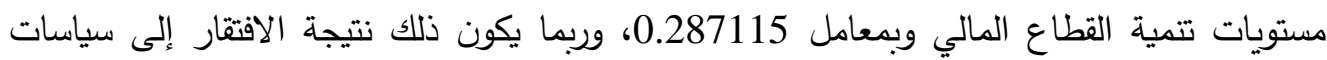

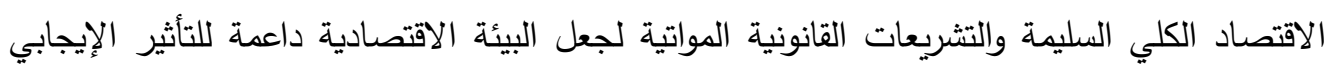
لمعدل نمو الناتج المحلي الإجمالي على تتمية القطاع المالي. (Benyah, 2010, 19) . Huang(2006) يثير الأثر السلبي لمؤشر الانفتاح التجاري (TRA) في مؤشر التتمية المالية (SPV) المناقض للنظرية

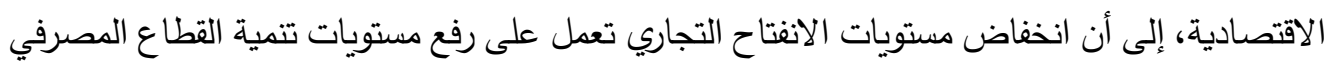

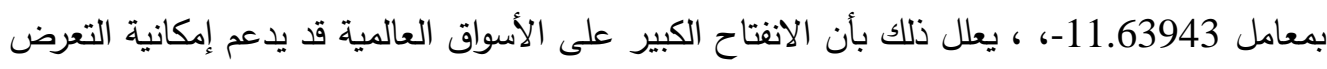

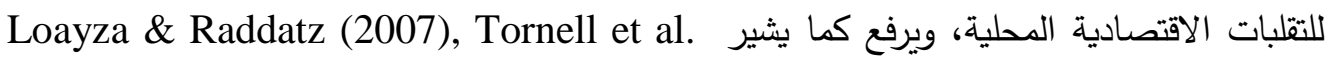
(2004) من مستويات ضعف الدولة أمام الصدمات الخارجية، الأمر الذي يعيق من تتمية القطاع المالي ع عيلي ويمنع تطوره (Kim,2010,254-261)

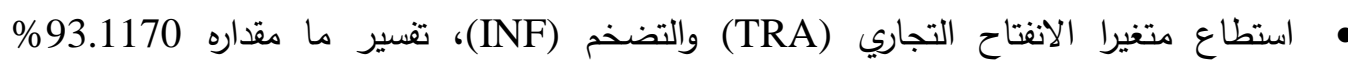
0.72814\%من التغيرات في الحاصلة في مؤشرات التتمية المالية (IS), (TD),على التوالي. في حين فسر متغيرا النمو الاقتصادي (GRO) والتضخم (INF) ما مقداره في الحاصلة في مؤشر التنمية المالية (BC). إلا أن متغير الانفتاح التجاري (TRA)، فسر ما مقداره 0.76994 \% من من التغيرات في الحاصلة في مؤشر التتمية المالية (SPV). فثل متغير الانفتاح التجاري (TRA) في إثبات تأثيره المعنوي في الأنموذج الأول لمؤشر التمية المالية في بلدان مجلس التعاون الخليجي، وربما يعود ذلك في جزء كبير منه إلى افتقار البلدان النامية

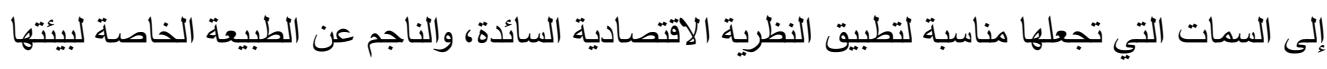
السياسية والاقتصادية والاجتماعية (العادات والتقاليد) والدينية (كالمبادئ الدينية الإسلامية التي تؤكد

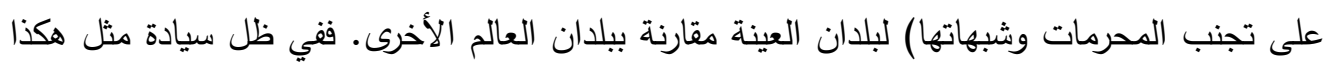




\section{أثر بعض متغيرات الاقتصاد الكلي في تنمية القطاع ...............}

الدباغ والعراقي

عوامل عشوائية غير قابلة للقياس قد تؤدي إلى فقدان بعض المتغيرات الاقتصادية تأثيرها الإيجابي الذي من المقرر وبحسب المنطق الاقتصادي أن تمارسه في تتمية القطاع المالي.

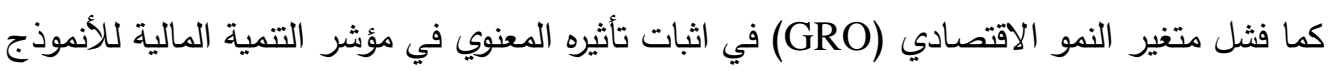
الثاني والثالث والرابع في بلدان مجلس التعاون الخليجي، ربما جاء ذلك نتيجة لفقدان العلاقة المباشرة بينه وبين تتمية القطاع المالي، فالمستويات المرتفعة منه يمكن أن تمارس تأثيرها في مؤشرات التتمية

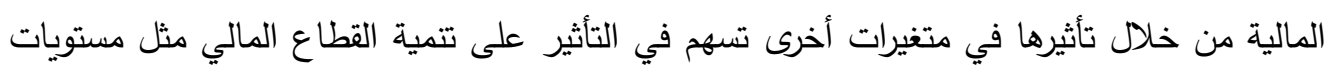
البنية التحتية الاقتصادية والمالية والمؤسساتية وغيرها.

\section{צ. الخاتمة Conclusion}

شكلت دراسة العوامل وتثخيصها التي يمكن أن تمارس تأثيرها في تتمية القطاع المالي بشقيه

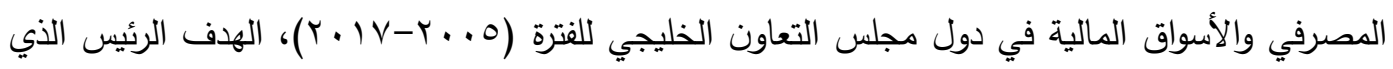

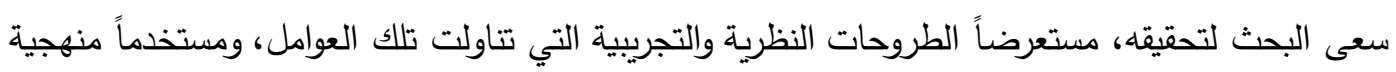
البيانات المزدوجة المتوازنة (Balanced Panel Data) في إثبات ذللك، وقد أفصحت نتائج البحث عن تعدد العوامل المؤثرة في تتمية القطاع المالي، فضلاً عن تباين طبيعة واتجاه وحجم تأثير تلك المتغيرات في تتمية

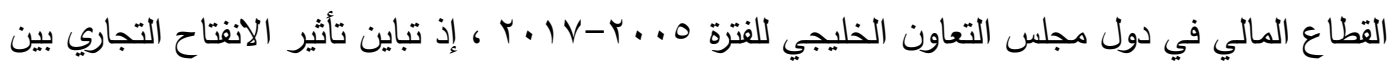

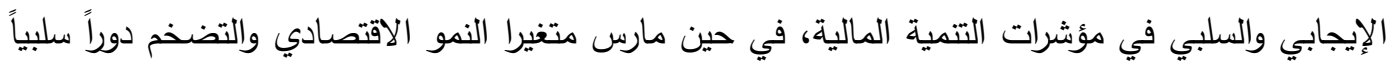

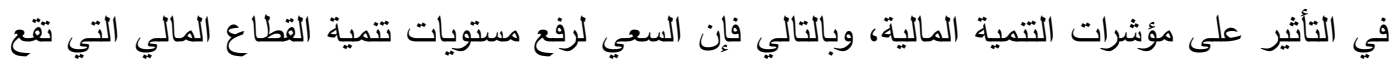

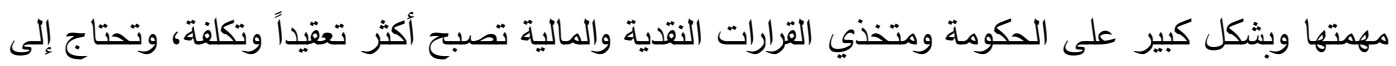
مديات زمنية طويلة نسبياً. فرفع كفاءة أداء البنية التحتية الاقتصادية والمالية والمؤسساتية التي توفير البيئة

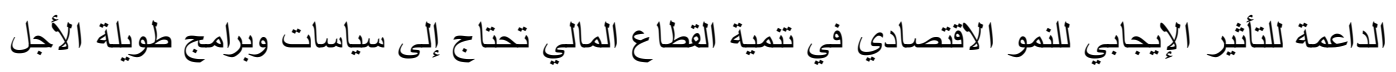
تقوم بتنفيذها الحكومة، فضلا عن ضرورة دعم ومساعدة المنظمات الدولية في الجوانب الفنية والمالية.

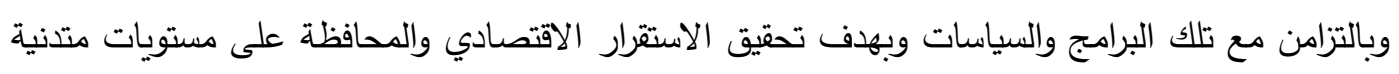

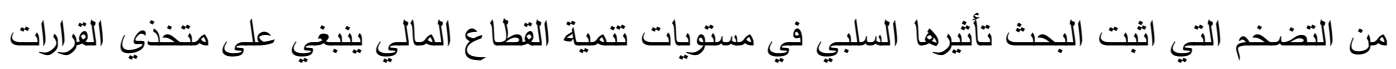
المالية والسلطة النقدية العمل على وضع وصياغة السياسات الكفيلة بمكافحة التضخم، من جانب آخر ، ينبغي لتبني

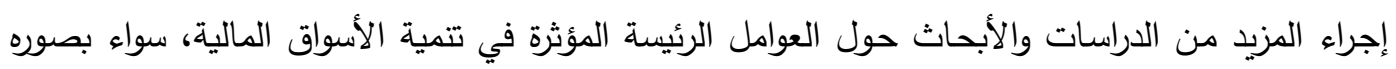

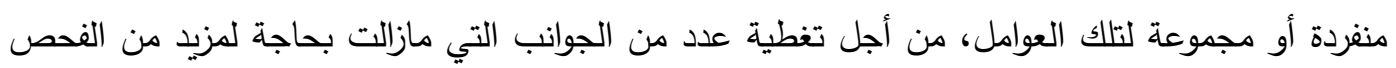
والتدقيق ومنها السياسية، والقانونية، والمؤسساتية، والاجتماعية.

\section{(1) الملحق}

التوصيف الاحصائي لمتغيرات الدراسة

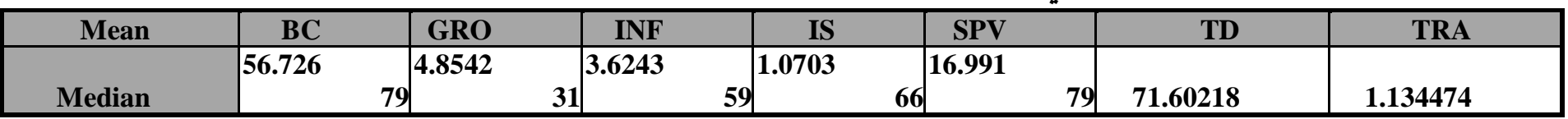


أثر بعض متغيرات الاقتصاد الكلي في تنمية القطاع

الدباغ والعراقي

\begin{tabular}{|c|c|c|c|c|c|c|c|}
\hline Maximum & $\begin{array}{|ll|}55.830 & \\
& 00 \\
\end{array}$ & 4.3600 & 2.9000 & 0.4052 & 14.545 & 76.34500 & 0.988750 \\
\hline Minimum & 105.19 & 26.170 & 15.100 & 8.7936 & 46.100 & 90.47000 & 1.918800 \\
\hline Std. Dev. & 30.650 & $\begin{array}{l}- \\
7.080000\end{array}$ & \begin{tabular}{|c|}
- \\
4.900000 \\
\end{tabular} & \begin{tabular}{|c|}
2.000000 \\
\end{tabular} & 7.0300 & 44.09000 & 0.618600 \\
\hline Skewness & 17.618 & $\begin{array}{|ll|}5.1267 & \\
& \end{array}$ & $\begin{array}{|ll|}3.5697 & \\
& 57 \\
\end{array}$ & \begin{tabular}{|l|l|}
1.8902 & \\
& 52 \\
\end{tabular} & 8.9086 & 12.14772 & 0.321578 \\
\hline Kurtosis & 0.5284 & 1.3251 & 1.2839 & 1.6581 & 1.1982 & -0.524391 & 0.822848 \\
\hline & 78 & 7.0573 & 4.9611 & 6.5665 & 3.9601 & 2.001898 & 2.544614 \\
\hline $\begin{array}{ll}\text { Jarque- } & \\
& \text { Bera } \\
\end{array}$ & & & & & & & \\
\hline Probability & 75 & 76.330 & 33.931 & 77.082 & 21.662 & 6.812496 & 9.475992 \\
\hline & 0.1236 & 0.0000 & 0.0000 & 0.0000 & 0.0000 & 0.033165 & 0.008756 \\
\hline Sum & & & & & & & \\
\hline $\begin{array}{lll}\text { Sum } & \text { Sq. } & \\
& & \text { Dev. } \\
\end{array}$ & 4424.6 & 378.63 & 282.70 & 83.488 & 1325.3 & 5584.970 & 88.48900 \\
\hline & $\begin{array}{|ll|}23901 . & \\
& 88 \\
\end{array}$ & 2023.8 & 981.22 & 275.12 & 6111.0 & 11362.67 & 7.962756 \\
\hline Observatio & & & & & & & \\
\hline Mean & 78 & 78 & 78 & 78 & 78 & 78 & 78 \\
\hline
\end{tabular}

الجدول من عمل الباحثين بالاعتماد على مخرجات برنامج Eviews10

TANMIYAT AL-RAFIDAIN (P-ISSN: 1609-591X; E-ISSN: 2664-276X) تنمية الرافدين 
(2)
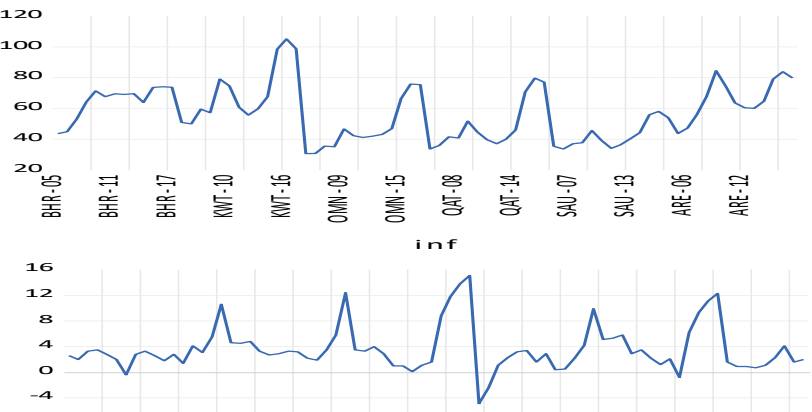

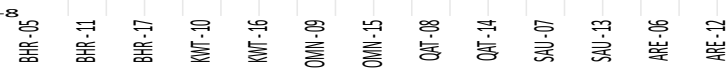

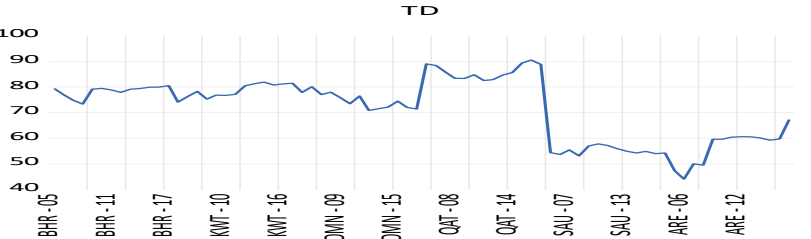

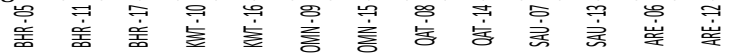

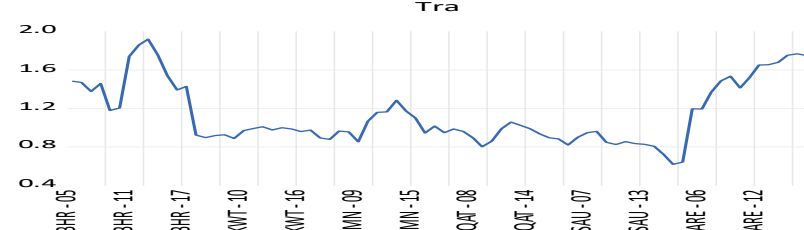

الرسوم البيانية للبيانات المزدوجة لمتغيرات الاراسة وبصيغتها الخطية

Eviews الثكل من إعداد الباحثين بالاعتماد على نتائج برنامج 10 •

(3) الملحق (3)

نتائج اختبار Levin-Lin-Chu,2002 لاستقراريه البيانات المزدوجة Panel Data لمتغيرات

الاراسة بصيغتها الخطية

\begin{tabular}{|c|c|c|c|c|c|c|}
\hline \multirow[b]{2}{*}{ Variables } & \multicolumn{3}{|c|}{ Level } & \multicolumn{3}{|c|}{ First Difference } \\
\hline & None & $\begin{array}{c}\text { Intercep } \\
\mathbf{t}\end{array}$ & $\begin{array}{l}\text { Trend and } \\
\text { Intercept }\end{array}$ & None & Intercept & $\begin{array}{l}\text { Trend and } \\
\text { Intercept }\end{array}$ \\
\hline $\begin{array}{c}\text { BC } \\
\text { Prob. }\end{array}$ & $\begin{array}{c}2.69388 \\
0 \\
0.9965 \\
\end{array}$ & $\begin{array}{c}-0.62908 \\
0.2646\end{array}$ & $\begin{array}{c}-1.66411 \\
0.0480\end{array}$ & $\begin{array}{c}-5.75349 \\
0.0000\end{array}$ & $\begin{array}{c}-4.20746 \\
0.0000\end{array}$ & $\begin{array}{c}-1.95565 \\
0.0253\end{array}$ \\
\hline $\begin{array}{l}\text { GRO } \\
\text { Prob. }\end{array}$ & $\begin{array}{c}-\overline{3.34875} \\
0.0004 \\
\end{array}$ & $\begin{array}{c}-4.53562 \\
0.0000\end{array}$ & $\begin{array}{c}-7.31091 \\
0.0000\end{array}$ & & & \\
\hline $\begin{array}{l}\text { INF } \\
\text { Prob. }\end{array}$ & $\begin{array}{c}2.95095 \\
0.0016\end{array}$ & $\begin{array}{c}-2.57692 \\
0.0050\end{array}$ & $\begin{array}{c}-4.02401 \\
0.0000\end{array}$ & & & \\
\hline $\begin{array}{c}\text { IS } \\
\text { Prob. }\end{array}$ & $\begin{array}{c}- \\
5.17765\end{array}$ & $\begin{array}{c}-6.94721 \\
0.0000\end{array}$ & $\begin{array}{c}-5.37916 \\
0.0000\end{array}$ & & & \\
\hline
\end{tabular}

TANMIYAT AL-RAFIDAIN (P-ISSN: 1609-591X; E-ISSN: 2664-276X) تنمية الرافدين 
أثر بعض متغيرات الاقتصاد الكلي في تنمية القطاع ..

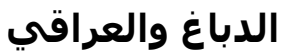

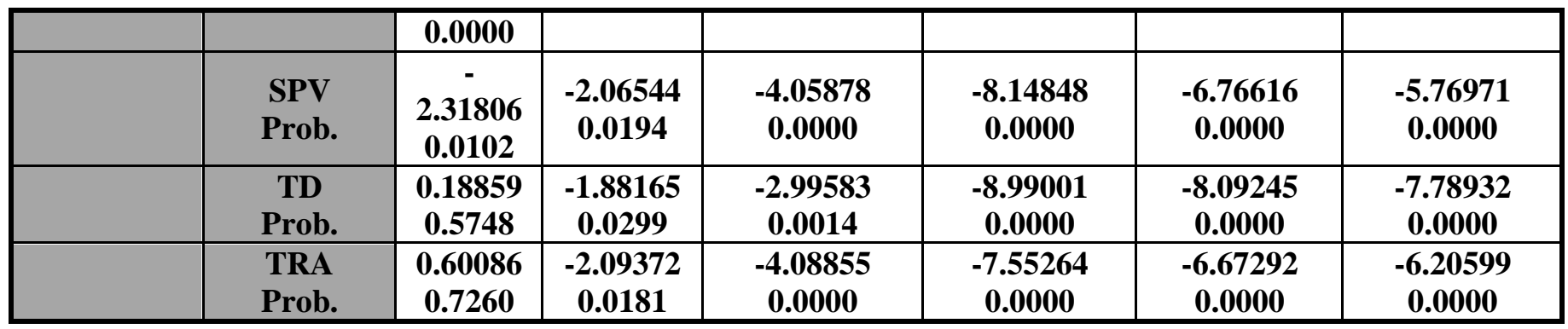

Eviews10 الجدول من عمل الباحثين بالاعتماد على مخرجات برنامج •

• • القيم في الجدول تمثل قيم Statistic t.

• اختيار فترة الابطاء تم اعتماد Automatic selection of maximum lags ضمن برنامج

Eviews 10

\section{Refrence}

Ake ,Boubakari Ake\& Ognaligui ,Rachelle Wouono, 2010, Financial Stock Market and Economic Growth in Developing Countries: The Case of Douala Stock Exchange in Cameroon, International Journal of Business and Management, 5(5):82-88.

Al-Iraqi, Bashar, 2017, The Impact of Money Supply Fluctuations on Stock Prices: Developing a Model for Financial Markets in the Gulf Cooperation Council Countries, (24) 3, 445-474, Arab Journal of Management Sciences.

Al-Iraqi, Bashar, 2018, Factors Determining the Revenues of Monetary Issue in the Arab Countries, Journal of Development and Economic Policies, (20) 1, 71-104.

Al-Omari, Muhammad Walid, Al-Hait, Nahil Ismail Saqf, 2016, The Impact of Technical Efficiency and Exports on the Profitability of the Jordanian Pharmaceutical Industry Enterprises, The Jordanian Journal of Business Administration, (12) 4, 877-898.

Aluko, Olufemi A. \& Ajayi, Michael Adebayo 2018,Determinants of banking sector development: Evidence from Sub-Saharan African countries, Borsa Istanbul Review , 18(2),122-139

Ardicad, Oya Pinar \& Damar, H. Evren,2006, Financial Sector Deepening and Economic Growth: Evidence From Turkey, MPRA Paper 4077,University Library of Munich, Germany.

Arestis ,Philip \& Demetriades ,Panicos ,1996,Finance and growth: Institutional Considerations and Causality, UEL, Department of Economics Working Paper, No.5

Arestis,Philip \& Demetriades, Panicos,1997, Financial Development and Economic Growth: Assessing the Evidence, Wiley, The Economic Journal, Vol. 107, No. 442 (May, 1997), pp. 783-799.

Asiama, Johnson P\& Mobolaji, Hakeem,2011, Trade and Financial Openness, Institutional Quality, and Financial Development in Sub-Sahara Africa (SSA), Nigeria.

TANMIYAT AL-RAFIDAIN (P-ISSN: 1609-591X; E-ISSN: 2664-276X) تنمية الرافدين 
Badeeb, Ramez Abubakr \& Lean, Hooi Hooi, 2017, The Determinants of Financial Development in the Republic of Yemen: Evidence from the Principal Components Approach, Capital Markets Review Vol. 25, No. 2, pp. 32-48.

Balogun ,Wakilat Olabisi , Asso ,Jauhari. B.\& Hassan,Sallahuddin B. ,2016, Long Term Effect of Liquidity on Stock Market Development,Journal of Economics and Sustainable Development Vol.7, No.4

Baltagi, Badi H., Demetriades, Panicos O., \& Law, Siong H. (2009). Financial development and openness: Evidence from panel data. Journal of Development Economics, 89(2), 285-296

Baum, Christopher F., Caglayan, Mustafa \& Ozkan, Neslihan (2009). The second moments matter: The impact of macroeconomic uncertainty on the allocation of loanable funds. Economics Letters, 102(2):87-89

Baum, Christopher F.,et al. (2006). The impact of macroeconomic uncertainty on non-financial firms' demand for liquidity. Review of Financial Economics, 15():289-304.

Bayar, Y1lmaz, Gündüz ,Murat\& Sezgin, Funda H. , 2019, Banking Sector Instability and Economic Growth: Evidence from Turkey, alphanumeric journal, (7)2, 264-274.

Beck, Thorsten (2002). Financial development and international trade: Is there a link?. Journal of International Economics, 57(1), 107-131

Ben Naceur ,Sami, Cherif, Mondher \& Kandil, Magda ,2014 What drives the development of the MENA financial sector?,Borsa _Istanbul Review 144,212-223.

Benyah, Francella Ewurama Ketsina, 2010, The Determinants of Financial Development: A Focus on African Countries, Master Thesis in Economics.Jonkoping university.

Bittencourt, Manoel (2012). Financial development and economic growth in Latin America: Is Shumpeter right? Journal of Policy Modeling, 34(3):341-355

Bittencourt, Manoel,2008, Inflation and Financial Development: Evidence from Brazil, United Nation University -WIDER, Research Paper No.14

Boyd,John H., Levine, Ross\& Smith,Bruce D.,2001, The impact of inflation on financial sector performance, Journal of Monetary Economics, Volume 47, Issue 2, Pages 221-248.

Calderón, Cesar Augusto Valbuena \& Liu, Lin, 2003, The Direction of Causality Between Financial Development and Economic Growth, Journal of Development Economics 72(1):321-334 .

Christopoulos, Dimitris \& Tsionas ,Efthymios Mike, 2004, Financial development and economic growth: Evidence from panel unit root and cointegration tests, Journal of Development Economics 73(1):55-74.

Čihák, Martin et al.,2012, Financial Development in 205 Economies, 1960 to 2010, NBER Working Paper No. 18946, Cambridge.

Clark, Paul, Crawford, Claire, Steele, Fiona \& Vignoles, Anna, 2010, The Choice Between Fixed and Random Effects Models: Some Considerations For

TANMIYAT AL-RAFIDAIN (P-ISSN: 1609-591X; E-ISSN: 2664-276X) تنمية الرافدين 


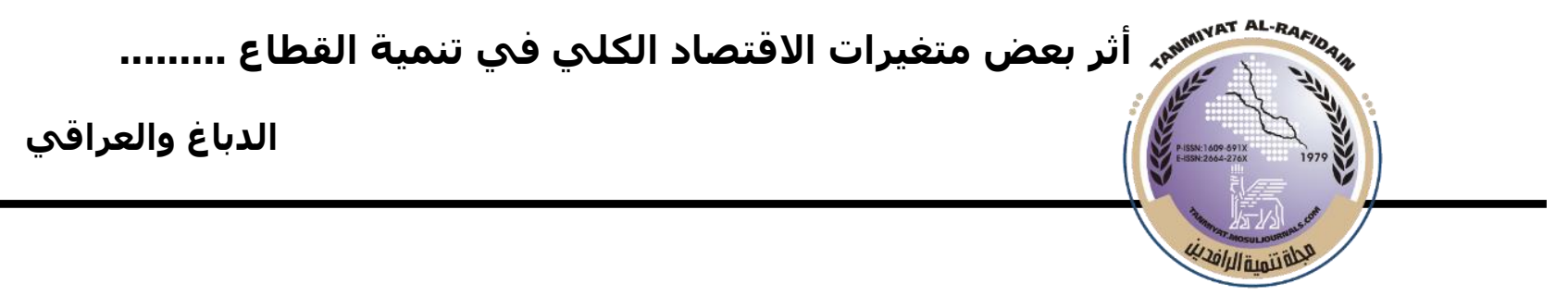

Educational Research, Centre for Market and Public Organisation Bristol Institute of Public Affairs University of Bristol, Working Paper No. 10/240.

DeCoursey, W.J., 2003, Statistics and Probability for Engineering Applications With Microsoft Excel, Newnes is an imprint of Elsevier Science.

Demetriades, Panicos O. \& Hussein, Khaled A. 1996, Does financial development cause economic growth? Time-series evidence from 16 countries, Journal of Development Economics.51(2): 387-411

Demirguc-Kunt , Asli \& Levine, Ross,1996, Stock market development and financial intermediaries: stylized facts, World Bank Economic Review, 10(2): 291-321.

Didier, Tatiana \& Schmukler, Sergio L. (2014). Emerging Issues in Financial Development. Lessons from Latin America. Washington, DC: The World Bank.

Do, Quy-Toan \& Levchenko ,Andrei A.,2004, Trade and Financial Development, World Bank Policy Research ,Working Paper No.3347,30 Pages.

Ehigiamusoe, Kizito Uyi, Guptan, Vinitha, \& Narayanan, Suresh,2019, The effects of income and inflation on financial development: Evidence from heterogeneous panels, Economics Discussion Papers, No. 2019-11.

Ghali, Khalifa H. ,1999, Financial Development and Economic Growth: The Tunisian Experience, Review of Development Economics,3(3): 310-322.

Gil-García, J. Ramón \& Puron-Cid, y Gabriel Puron-Cid, 2013, Using Panel Data Techniques for Social Science Research: an Illustrative Case and Some Guidelines, C I E N C I A e r g o -s um , (21)3, 203-216.

Gozgor, Giray (2015). Causal relation between economic growth and domestic credit in the economic globalization: Evidence from the Hatemi-J's test. The Journal of International Trade and Economic Development, 24(3), 395-40

Greene, William H., 2002, Econometric Analysis, fifth edition, New York University.

Gregorio, Jose De \& Guidotti, Pablo E.,1995, Financial development and economic growth, World Development, 23(3):433-448.

Gujarati, Damodar N., 2004, Basic Econometrics, Fourth Edition, The McGraw-Hill Companies.

Gupta, Gaurav \& Mahakud, Jitendram,2019, Alternative measure of financial development and investment-cash flow sensitivity: evidence from an emerging Economy, Financial Innovation, vol. 5, Article No. 1:P.9

Haslag, Joseph H. \& Koo, Jahyeong (1999). Financial Repression, Financial Development and Economic Growth. Working Papers 9902 Federal Reserve Bank of Dallas

Hayek, Mohammad Ali Al Hayek, 2018, The Relationship between Revenues Size and Actual Expenditures through the Closing Account Result of the Jordanian Government, International Journal of Business and Management; (13) 2, 40-52.

TANMIYAT AL-RAFIDAIN (P-ISSN: 1609-591X; E-ISSN: 2664-276X) تنمية الرافدين 
Ho, Sin-Yu \& Bernard, Njindan Iyke, 2018, Short- and Long-term Impact of Trade Openness on Financial Development in Sub-Saharan Africa, MPRA Paper No. 84272 .

Huang, Yongfu,2010,Determinants of Financial Development, University of Cambridge.Index of Financial Development, IMF Working Paper.No.5.

Hurlin, Christophe \& Venet, Baptiste (2008) Financial Development and Growth: A Re-examination using a panel Granger Causality Test, hal archivesouvertes.fr.

Huybens, Elisabeth, Smith, Bruce D. (1999) Inflation, financial markets, and longrun real activity. Journal of Monetary Economics, 43(2), 283-315

Khan ,Mohsin S. \& Senhadji ,Abdelhak S. ,2003, Financial Development and Economic Growth: A Review and New Evidence, Journal of African Economies, (12)2, 89-110

Kim, Dong-Hyeon, Lin, Shu-Chin \& Suen, Yu-Bo,2010, Dynamic effects of trade openness on financial development, Economic Modelling, 27: 254-261

King , Robert G.\& Levine, Ross, 1993, Finance, entrepreneurship, and growth Theory and evidence, Journal of Monetary Economics 32 (1993) 513-542. North-Holland.

Law, Siong Hook \& Habibullah, Muzafar Shah (2009). The determinants of financial development: Institutions, openness and financial liberalisation. South African Journal of Economics, 77(1), 45-58

Levine ,Ross, Loayza, Norman \& Beck, Thorsten ,2000, Financial intermediation and growth: Causality and causes, Journal of Monetary Economics 46 .3177.

Levine Ross\& Zervos,Sara,1998,Stock Markets, Banks, and Economic Growth ,The American Economic Review, Vol. 88, No.3, pp. 537-558.

Nazir ,Mian Sajid , Nawaz ,Muhammad Musarat \& Gilani ,Usman Javed ,2010, Relationship between economic growth and stock market development, African Journal of Business Management Vol. 4(16), pp. 3473-3479.

Nejad, Omid Dehghan, 2010, Determinants of Financial Development in Iran: Do Financial Repression Policies Hinder Financial Development?, Degree of Master of Science, Institute of Graduate Studies and Research, Eastern Mediterranean University, Gazimağusa, North Cyprus

Newbery, David. M., \& Stiglitz, Joseph. E. (1984). Pareto inferior trade. The Review of Economic Studies, 51(1), 1-12

Odhiambo ,Nicholas M ,2012, The Impact Of Inflation On Financial Sector Development: Experience From Zambia, Journal of Applied Business Research 28(6):1497-1508.

Ozturk, Nurettin \& Karagoz, Kadir,2012, Relationship Between Inflation and Financial Development: Evidence from Turkey, International Journal of Alanya Faculty of Businessm,4(2): 81-87

Ozturk, Nurettin \& Karagoz, Kadir,2012, Relationship Between Inflation and Financial Development: Evidence from Turkey, International Journal of Alanya Faculty of Businessm,4(2): 81-87

TANMIYAT AL-RAFIDAIN (P-ISSN: 1609-591X; E-ISSN: 2664-276X) تنمية الرافدين 
Peia, Oana \& Roszbach, Kasper (2015). Finance and growth: time series evidence on causality. Journal of Financial Stability, 19, 105-118.

Rajan, Raghuram G. \& Zingales, Luigi,2003, The great reversals: the politics of financial development in the twentieth century, Journal of Financial Economics 69 (2003) 5-50.

Sukcharoensin, Pariyada and Sukcharoensin, Sorasart,2013, The Analysis of Stock Market Development Indicators: Evidence from the ASEAN-5 Equity Markets, International Journal of Trade, Economics and Finance, 4(6):343346.

Svaleryd, Helena, \& Vlachos, Jonas. (2002). Markets for risk and openness to trade: how are they related?. Journal of International Economics, 57(2), 369395.

Svaleryd, Helena., \& Vlachos, Jonas. (2005). Financial markets, the pattern of industrial specialization and comparative advantage: Evidence from OECD countries. European Economic Review, 49(1), 113-144.

Svirydzenka ,Katsiaryna,2016,Strategy, Policy, and Review Department Introducing a New Broad-based Index of Financial Development, International Monetary Fund WP/16/5 IMF Working Paper

Takyi Paul Owusu \& Obeng, Camara Kwesi, 2013, Determinants of financial development in Ghana, International Journal of Development and Sustainability, Volume 2 Number 4,1-13.

$\mathrm{Xu}$, Zhenhui, 2000, Financial development, investment, and economic growth, Economic Inpoury,38(2).331-344.

Zang, Hyoungsoo \& Kim, Young Chul (2007). Does financial development precede growth? Robinson and Lucas might be right. Applied Economics Letters, 14(1), 15-19

Zermeñoa, Miguel Á. Tinoco, Martínezb, Francisco Venegas \& Preciadoc Víctor H. Torres, 2018, Effects of inflation on financial sector performance: New evidence from panel quantile regressions, Investigación Económica, 77(303):94-129. 